Preprint typeset in JHEP style - HYPER VERSION

OU-HEP-111006

\title{
Coupled Boltzmann calculation of mixed axion/neutralino cold dark matter production in the early universe
}

\author{
Howard Baer $^{a}$, Andre Lessa ${ }^{b}$ and Warintorn Sreethawong ${ }^{a}$ \\ ${ }^{a}$ Dept. of Physics and Astronomy, University of Oklahoma, Norman, OK 73019, USA \\ ${ }^{b}$ Instituto de Física, Universidade de São Paulo, São Paulo - SP, Brazil \\ E-mail: baer@nhn.ou.edu, lessa@fma.if.usp.br, wstan@nhn.ou.edu
}

\begin{abstract}
We calculate the relic abundance of mixed axion/neutralino cold dark matter which arises in $R$-parity conserving supersymmetric (SUSY) models wherein the strong $\mathrm{CP}$ problem is solved by the Peccei-Quinn (PQ) mechanism with a concommitant axion/saxion/axino supermultiplet. By numerically solving the coupled Boltzmann equations, we include the combined effects of 1 . thermal axino production with cascade decays to a neutralino LSP, 2. thermal saxion production and production via coherent oscillations along with cascade decays and entropy injection, 3. thermal neutralino production and re-annihilation after both axino and saxion decays, 4 . gravitino production and decay and 5. axion production both thermally and via oscillations. For SUSY models with too high a standard neutralino thermal abundance, we find the combined effect of SUSY PQ particles is not enough to lower the neutralino abundance down to its measured value, while at the same time respecting bounds on late-decaying neutral particles from BBN. However, models with a standard neutralino underabundance can now be allowed with either neutralino or axion domination of dark matter, and furthermore, these models can allow the $\mathrm{PQ}$ breaking scale $f_{a}$ to be pushed up into the $10^{14}-10^{15} \mathrm{GeV}$ range, which is where it is typically expected to be in string theory models.
\end{abstract}

KEYWORDs: Supersymmetry Phenomenology, Supersymmetric Standard Model, Dark Matter, Axions. 


\section{Introduction}

The Standard Model (SM) of particle physics is beset by two afflictions: 1. in the scalar (Higgs) sector of the theory, quadratic divergences require large fine-tunings of electroweak parameters which depend on the scale $\Lambda$ below which the SM is regarded as the correct effective field theory of nature and 2. in the QCD sector of the theory, the Lagrangian term

$$
\mathcal{L} \ni \frac{\bar{\theta}}{32 \pi^{2}} F_{A \mu \nu} \tilde{F}_{A}^{\mu \nu}
$$

required by 'tHooft's solution to the $U(1)_{A}$ problem is constrained to a value $\bar{\theta} \lesssim 10^{-10}$ to gain accord with measurements of the neutron EDM[1]. The first of these is solved by the introduction of softly broken weak scale supersymmetry (SUSY) into the theory 2] (which receives some indirect support from the measured values of gauge couplings at LEP [3] and from global fits to precision electroweak data[4]), while the second problem is solved by the introduction of a global $U(1)_{P Q}$ Peccei-Quinn (PQ) symmetry broken by QCD anomalies[5], which requires the existence of an ("invisible") axion [6, , ], with mass expected in the micro-eV or below range [8]. Solving both problems simultaneously requires supersymmetrization of the SM (usually via the Minimal Supersymmetric Standard Model, or MSSM) along with the introduction of an axion supermultiplet $\hat{a}$ into the theory. The $\hat{a}$ supermultiplet contains an $R$-parity-even spin-0 saxion field $s(x)$ along with an $R$-parityodd spin- $\frac{1}{2}$ axino $\tilde{a}(x)$, in addition to the usual pseudoscalar axion field $a(x)$ :

$$
\hat{a}=\frac{s+i a}{\sqrt{2}}+i \sqrt{2} \bar{\theta} \tilde{a}_{L}+i \bar{\theta} \theta_{L} \mathcal{F}_{a}
$$

in 4-component spinor notation[2].

In such a theory, it is expected that SM superpartner particles with weak scale masses should emerge, along with a weak scale saxion, whilst the axino mass is more model dependent, with $m_{\tilde{a}} \sim \mathrm{keV}-\mathrm{TeV}$ being expected[9]. The axion, saxion and axino couplings to matter depend on the PQ breaking scale $f_{a}{ }^{1}$, which is required $f_{a} \gtrsim 10^{9} \mathrm{GeV}$ by stellar cooling calculations 10]. The axion is often considered as a very appealing dark matter (DM) candidate 11, 12]. ${ }^{2}$

In the MSSM, DM candidates include the lightest neutralino $\widetilde{Z}_{1}$ (a WIMP), the spin- $\frac{3}{2}$ gravitino $\widetilde{G}$ or possibly the superpartner of a right-handed neutrino [14]. Gravitino dark matter is tightly constrained and disfavored by the standard picture of Big Bang nucleosynthesis (BBN) [15], whilst right-hand neutrino states are expected to exist near the GUT scale according to the elegant see-saw mechanism for neutrino mass[16]. Many authors thus expect dark matter to be comprised of the SUSY neutralinos, a natural WIMP candidate which is motivated by the so-called "WIMP miracle". However, detailed analyses show that neutralino dark matter requires a rather high degree of fine-tuning [17] to match the

\footnotetext{
${ }^{1}$ Throughout this work we omit the number of generations factor $N$, which appear along with the PQ scale, $f_{a} / N$, in the DSFZ model and in the KSVZ model with more than one heavy quark generation. All our results can then be trivially generalized replacing $f_{a}$ by $f_{a} / N$.

${ }^{2}$ For a somewhat different axion/axino scenario, see Ref. 13.
} 
WMAP-measured cold DM abundance 18:

$$
\Omega_{\mathrm{DM}} h^{2}=0.1123 \pm 0.0035 \text { at } 68 \% \mathrm{CL} .
$$

In fact, the measured abundance lies in the most improbable locus of values of neutralino relic density as predicted by general scans over SUSY model parameter space 19.

The PQ-extended Minimal Supersymmetric Standard Model (PQMSSM) offers additional possibilities to describe the dark matter content of the universe. In the PQMSSM, the axino may play the role of stable lightest SUSY partner (LSP) [20, 21], while the quasistable axion may also constitute a component of DM[22], giving rise to mixed axion/axino

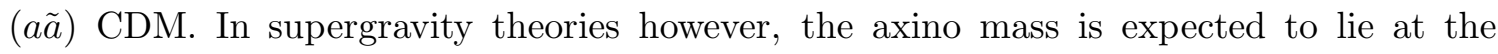
weak scale[23], so that the neutralino remains as LSP, and the possibility occurs for mixed axion/neutralino $\left(a \widetilde{Z}_{1}\right)$ CDM.

In a recent paper, Choi et al. 24. presented a semi-analytic approach for estimating the relic abundance of neutralinos in the mixed $a \widetilde{Z}_{1}$ CDM scenario. This approach applies to cases where the thermally averaged neutralino annihilation cross section times relative velocity $\langle\sigma v\rangle$ is approximately constant with temperature, as occurs for a wino-like or higgsino-like neutralino 25]. Detailed calculations of the relic abundance of mixed $a \widetilde{Z}_{1}$ CDM were performed in Ref. [26], where formulae for the neutralino and axion abundances were presented.

The standard calculation of the neutralino Yield $Y_{\widetilde{Z}_{1}}^{s t d} \equiv \frac{n_{\widetilde{Z}_{1}}}{s}$ (where $n_{\widetilde{Z}_{1}}$ is the neutralino number density and $s$ is the entropy density) gives

$$
Y_{\widetilde{Z}_{1}}^{s t d}=\frac{\left(90 / \pi^{2} g_{*}\left(T_{f r}\right)\right)^{1 / 2}}{4\langle\sigma v\rangle M_{P} T_{f r}},
$$

where $g_{*}\left(T_{f r}\right)$ is the number of active degrees of freedom at temperature $T=T_{f r}$, where

$$
T_{f r}^{s t d}=m_{\widetilde{Z}_{1}} / \ln \left[\frac{3 \sqrt{5}\langle\sigma v\rangle M_{P} m_{\widetilde{Z}_{1}}^{3 / 2}}{\pi^{5 / 2} T_{f r}^{1 / 2} g_{*}^{1 / 2}\left(T_{f r}\right)}\right] .
$$

is the freeze-out temperature and $M_{P}$ is the reduced Planck mass.

If instead axinos are thermally produced (TP) at a large rate at re-heat temperature $T_{R}$ after inflation, then they cascade decay to (stable) neutralinos at decay temperature

$$
T_{D}^{\tilde{a}}=\sqrt{\Gamma_{\tilde{a}} M_{P}} /\left(\pi^{2} g_{*}\left(T_{D}^{\tilde{a}}\right) / 90\right)^{1 / 4},
$$

and can boost the neutralino abundance. The late-time injection of neutralinos into the cosmic soup at temperatures $T_{D}^{\tilde{a}}<T_{f r}$ may cause a neutralino re-annihilation effect such that the neutralino Yield is instead given by 24, 26.

$$
\left.Y_{\widetilde{Z}_{1}}^{r e-a n n}\right|_{T=T_{D}^{\tilde{a}}} \simeq \frac{\left(90 / \pi^{2} g_{*}\left(T_{D}^{\tilde{a}}\right)\right)^{1 / 2}}{4\langle\sigma v\rangle M_{P} T_{D}^{\tilde{a}}} .
$$

Since $T_{D}^{\tilde{a}}$ is typically in the $\mathrm{MeV}-\mathrm{GeV}$ range, i.e. well below $T_{f r} \sim m_{\widetilde{Z}_{1}} / 20$, the neutralino abundance after re-annihilation can be highly enhanced relative to the standard cosmological picture. In addition, one must fold into the relic abundance the axion contribution 
arising from coherent axion field oscillations beginning at axion oscillation temperature $T_{a} \sim 1 \mathrm{GeV}$.

An additional complication comes from entropy production from axino decay after $T_{f r}$ (which may dilute the neutralino abundance) or after $T_{a}$ (which may dilute the axion abundance). This may occur in the case where axinos temporarily dominate the energy density of the universe. Depending on the PQ parameters of the PQMSSM model $\left(f_{a}, m_{\tilde{a}}\right.$, initial axion misalignment angle $\theta_{i}$ and $T_{R}$ ), the dark matter abundance may be either neutralino- or axion-dominated. In fact, cases may occur where the DM relic abundance is shared comparably between the two. In the latter case, it might be possible to directly detect relic neutralino WIMP particles as well as relic axions!

While the semi-analytic treatment of Ref's [24] and [26] provides a broad portrait of the mixed $a \widetilde{Z}_{1} \mathrm{CDM}$ picture, a number of important features have been neglected. These include the following.

- For bino-like neutralinos, $\langle\sigma v\rangle \sim a+b T^{2}$ where $a \sim 0$ since we mainly have $p$-wave annihilation cross sections. In this case, $\langle\sigma v\rangle$ is no longer independent of temperature, and the simple formulae 1.4 and 1.7 are no longer valid.

- In Ref's [24] and [26], the effects of saxion production and decay in the early universe are neglected. In fact, saxion thermal production or production via coherent oscillations (CO) [27], followed by late time saxion decay, may inject considerable entropy into the early universe, thus diluting all relics present at the saxion decay temperature $T_{D}^{s}$. Saxions may also add to the neutralino abundance via decays such as $s \rightarrow \tilde{g} \tilde{g}$, followed by gluino cascade decays. There exists the possibility of saxion and axino co-domination of the universe. In this case, there might be a second neutralino re-annihilation taking place at $T_{D}^{s}$.

- The treatments of [24] and [26] invoke the "sudden decay" approximation for latedecaying axinos, whereas in fact the decay process is a continuous one proceeding in time until the decaying species is highly depleted (all have decayed).

- The treatments of [24] and [26] largely ignore the effect of gravitino production and decay in the early universe.

To include the above effects into a calculation of the mixed $a \widetilde{Z}_{1}$ relic abundance, one must go beyond the semi-analytic treatment presented in Ref's [24, 26], and proceed with a full solution of the coupled Boltzmann equations which govern various abundances of neutralinos, axinos, axions, saxions, gravitinos and radiation.

Toward this end, in Sec. 2 we present a simplified set of coupled Boltzmann equations, which we use to calculate the relic abundance of mixed axion/neutralino dark matter. More details about the approximations made and each term present in our equations are discussed in Appendix A.

In Sec. 3, we present various numerical results for the mixed $a \widetilde{Z}_{1}$ CDM scenario using the full set of Boltzmann equations. We find that, even after the inclusion of the saxion field, adjusting the parameters of the PQMSSM can only increase the neutralino 
abundance, and not decrease it, while at the same time respecting bounds on late-decaying neutral particles from BBN. This result is the same as found in Refs. [24] and [26], but now corresponds to a much stronger statement, since the saxion entropy injection had been neglected in the previous works. Furthermore, our results also apply to models with bino-like neutralinos, which could not be studied in the semi-analytical framework used in Refs. [24] and [26].

Since the neutralino abundance can be only enhanced in the PQMSSM, in models such as mSUGRA, those points which are excluded by a standard overabundance of neutralinos are still excluded in the PQMSSM! This rather strong conclusion does depend on at least three assumptions: 1 . that thermal axino production rates are not suppressed by low-lying $P Q$-charged matter multiplets 28$]^{3}, 2$. that saxion decay is dominated by gluon and gluino pairs and 3. that the assumed saxion field strength $s(x) \equiv \theta_{s} f_{a}$ is of order the PQ-breaking scale $f_{a}$, i.e. that $\theta_{s} \sim 1$.

We also examine several cases with a standard underabundance of neutralino dark matter. In these cases, again the neutralino abundance is only increased (if BBN constraints are respected). Thus, adjustment of PQMSSM parameters can bring models with an underabundance of neutralinos into accord with the measured DM relic density. In these cases, the DM abundance tends to be neutralino-dominated. Also, in these cases, solutions exist where the PQ scale $f_{a}$ is either near its lower range, or where $f_{a}$ is much closer to $M_{G U T}$, with $f_{a} \sim 10^{14} \mathrm{GeV}$ typically allowed. This is much closer to the scale of $f_{a}$ which is thought to arise from string theory [31]. In Sec. 5, we present a summary and conclusions.

\section{Mixed axion/neutralino abundance from coupled Boltzmann equations}

Here, we present a brief description of our procedure to calculate the relic abundance of mixed $a \widetilde{Z}_{1}$ CDM in the PQMSSM. A more detailed discussion is left to Appendix A.

\subsection{Boltzmann equations}

The general Boltzmann equation for the number density of a particle species can be generically written as 32]:

$$
\dot{n}_{i}+3 H n_{i}=S_{i}-\frac{1}{\gamma_{i}} \Gamma_{i} n_{i}
$$

where $S_{i}$ represents a source term, $\Gamma_{i}$ is the decay width and $\gamma_{i}$ is the relativistic dilation factor to take into account the suppressed decays of relativistic particles. To describe the thermal production of a particle species $i$ as well as its decoupling from the radiation fluid and the non-thermal production coming from other particles decays, we include in $S_{i}$ the following terms:

$$
S_{i}=-\left[n_{i}^{2}-\left(n_{i}^{e q}(T)\right)^{2}\right]\langle\sigma v\rangle_{i}(T)+\sum_{j} B R(j, i) \Gamma_{j} \frac{n_{j}}{\gamma_{j}}
$$

\footnotetext{
${ }^{3}$ Here, we assume standard rates for thermal axino production as calculated in the literature 21, 29, 30. In Ref. [28], it has been shown that if $P Q$-charged matter multiplets $\hat{\Phi}$ exist well below the PQ breaking scale $f_{a}$, then axino production is suppressed by factors of $m_{\Phi} / T_{R}$.
} 
where $\langle\sigma v\rangle$ is the (temperature dependent) thermally averaged annihilation cross section times velocity for the particle species $i, n_{i}^{e q}$ is its equilibrium number density and $B R(j, i)$ is the branching fraction for particle $j$ to decay to particle $i^{4}$

The Boltzmann equation then becomes:

$$
\dot{n}_{i}+3 H n_{i}=-\Gamma_{i} m_{i} \frac{n_{i}^{2}}{\rho_{i}}+\left[\left(n_{i}^{e q}(T)\right)^{2}-n_{i}^{2}\right]\langle\sigma v\rangle_{i}+\sum_{j} B R(j, i) \Gamma_{j} m_{j} \frac{n_{j}^{2}}{\rho_{j}}
$$

where we have used $\gamma_{i}=\rho_{i} / m_{i} n_{i}$. As discussed in Appendix A, the above equation is also valid for coherent oscillating fields once we take $B R(j, i)=0$ and $\langle\sigma v\rangle_{i}=0$.

It is also convenient to write an equation for the evolution of entropy:

$$
\begin{aligned}
\dot{S} & =\left(\frac{2 \pi^{2}}{45} g_{*}(T) \frac{1}{S}\right)^{1 / 3} R^{4} \sum_{i} B R(i, X) \frac{1}{\gamma_{i}} \Gamma_{i} \rho_{i} \\
\text { or } \quad \dot{S} & =\frac{R^{3}}{T} \sum_{i} B R(i, X) \Gamma_{i} m_{i} n_{i}
\end{aligned}
$$

where $B R(i, X)$ is the fraction of energy injected in the thermal bath from $i$ decays.

Along with Friedmann's equation,

$$
H=\frac{1}{R} \frac{d R}{d t}=\sqrt{\frac{\rho_{T}}{3 M_{P}^{2}}}, \text { with } \rho_{T} \equiv \sum_{i} \rho_{i}+\frac{\pi^{2}}{30} g_{*}(T) T^{4},
$$

the set of coupled differential equations, Eq's. 2.3, 2.4 and 2.5, can be solved as a function of time. More details on the solution of the above equations and the expressions used for $\langle\sigma v\rangle_{i}, B R(i, j)$ and $B R(i, X)$ are presented in Appendix $\mathrm{A}$.

\subsection{Present day abundances and constraints from BBN}

To compute the relic density of neutralinos and axions we evolve the various particle and sparticle abundances from $T=T_{R}$ until the final temperature $T_{F}$ is reached at which all unstable particles (save the axion itself) have decayed. The relic densities of the various dark matter species labeled by $i$ are then given by:

$$
\Omega_{i} h^{2}=\frac{\rho_{i}\left(T_{F}\right)}{s\left(T_{F}\right)} \times \frac{s\left(T_{C M B}\right)}{\rho_{c} / h^{2}} .
$$

In our calculations, a critical constraint comes from maintaining the success of the standard picture of Big Bang nucleosynthesis. Constraints from BBN on late decaying neutral particles (labeled $X$ ) have been calculated recently by several groups 34, 35, 36] (we explicitly use the results of Ref. [36]) and are presented as functions of 1 . the decaying

\footnotetext{
${ }^{4}$ In this paper, $i$ is summed over 1 . neutralinos $\widetilde{Z}_{1}, 2$. TP axinos $\tilde{a}, 3$. and 4. CO- and TP-produced saxions $s(x), 5$. and 6 . CO- and TP-axions $a, 7$. TP gravitinos $\widetilde{G}$ and radiation. We allow for axino decay to $g \tilde{g}, \gamma \widetilde{Z}_{i}$ and $Z \widetilde{Z}_{i}$ states $(i=1-4)$, and saxion decay to $g g$ and $\tilde{g} \tilde{g}$. Additional model-dependent saxion decays e.g. to $a a$ and/or $h h$ are possible and would modify our results. We assume $\widetilde{G}$ decay to all particle-sparticle pairs, and include 3-body gravitino modes as well 33].
} 
neutral particle's hadronic branching fraction $B_{h}, 2$. the decaying particle's lifetime $\tau_{X}$, and 3. the decaying particle's relic abundance $\Omega_{X} h^{2}$ had it not decayed. The constraints also depend on 4 . the decaying particle's mass $m_{X}$. We have constructed digitized fits to the constraints given in Ref. [36], and apply these to late decaying gravitinos, axinos and saxions. Typically, unstable neutrals with decay temperature below $5 \mathrm{MeV}$ (decaying during or after $\mathrm{BBN}$ ) and/or large abundances will be more likely to destroy the predicted light element abundances.

\subsection{Example: calculation from a generic mSUGRA point}

As an example calculation, we adopt a benchmark point from the paradigm minimal supergravity model (mSUGRA), with parameters $\left(m_{0}, m_{1 / 2}, A_{0}, \tan \beta, \operatorname{sign}(\mu)\right)=(400 \mathrm{GeV}$, $400 \mathrm{GeV}, 0,10,+)$. The sparticle mass spectrum is generated by Isasugra 37], and has a bino-like neutralino with mass $m_{\widetilde{Z}_{1}}=162.9 \mathrm{GeV}$ and a standard relic abundance from IsaReD 38] of $\Omega_{\widetilde{Z}_{1}}^{\text {std }} h^{2}=1.9$ (it would thus be excluded by WMAP7 measurements assuming the standard neutralino freeze-out calculation). We assume a gravitino mass $m_{\widetilde{G}}=1 \mathrm{TeV}$.

Here, we work in the PQMSSM framework, and take $T_{R}=10^{10} \mathrm{GeV}$ with PQ parameters as $m_{\tilde{a}}=1 \mathrm{TeV}, m_{s}=5 \mathrm{TeV}, \theta_{i}=0.5$ and $f_{a}=10^{12} \mathrm{GeV}$. We also take $\theta_{s}=1$, where $\theta_{s} f_{a}$ is the initial field amplitude for coherent oscillating saxions. The various energy densities $\rho_{i}$ are shown in Fig. 目 for $i=R$ (radiation), $\widetilde{Z}_{1}$ (neutralinos), $a^{T P}$ (thermally produced axions), $a^{C O}$ (coherent oscillating axions), $s^{T P}$ (thermally produced saxions), $s^{C O}$ (coherent oscillating saxions), $\tilde{a}^{T P}$ (thermally produced axinos) and $\widetilde{G}^{T P}$ (thermally produced gravitinos). The energy densities are plotted against scale factor ratio $R / R_{0}$, where $R_{0}$ is the scale factor at $T=T_{R}$. We also plot the temperature $T$ of radiation (green-dashed curve).

We see that, at $R / R_{0}<10^{10}$, the universe is indeed radiation-dominated. At $T \gg 1$ $\mathrm{TeV}$, the TP axions, saxions and axinos all have similar abundances. At these temperatures, the saxion coherent abundance as well as the gravitino thermal abundance are far below the other components. As the universe expands and cools, most components are relativistic, and decrease with the same slope as radiation: $\rho_{i} \sim T^{-4}$. The exception is the COproduced saxions, which are non-relativistic, and fall-off as $\rho_{s}^{C O} \sim T^{-3}$. At $R / R_{0} \sim 10^{7}$, the temperature $T \sim 1 \mathrm{TeV}$, and the thermally-produced axinos, saxions and gravitinos become non-relativistic, so now $\rho_{\tilde{a}, s, \widetilde{G}}^{T P} \sim T^{-3}$. For even lower temperatures with $R / R_{0} \sim 10^{9}$, neutralinos begin to freeze-out, and their abundance falls steeply. At $T \sim m_{\widetilde{Z}_{1}} / 20$, they do freeze-out, and normally their density would fall as $\rho_{\widetilde{Z}_{1}} \sim T^{-3}$, as indicated by the blue dotdashed curve, which shows neutralino abundance in the MSSM, without PQ-augmentation. In the PQMSSM however, saxions- and later still axinos- begin decaying in earnest, and feed into the neutralino abundance, preventing its usual fall as $T^{-3}$. At $T \sim 0.5 \mathrm{GeV}$, the energy density of axinos surpass the radiation component and the universe becomes axino-dominated until the axino decays at $T \sim 10 \mathrm{MeV}$. Also, around $R / R_{0} \sim 3 \times 10^{10}$ with $T \sim 1 \mathrm{GeV}$, CO production of axions begins, and by $R / R_{0} \sim 4 \times 10^{11}$, with $T \lesssim \Lambda_{Q C D}$, its abundance begins to fall as $T^{-3}$. For even lower temperatures $(T<10 \mathrm{MeV})$, the axinos have essentially all decayed, feeding back into the neutralino abundance, and also increasing the entropy per co-moving volume, which would otherwise be conserved. At $R / R_{0} \sim 10^{14}$, 


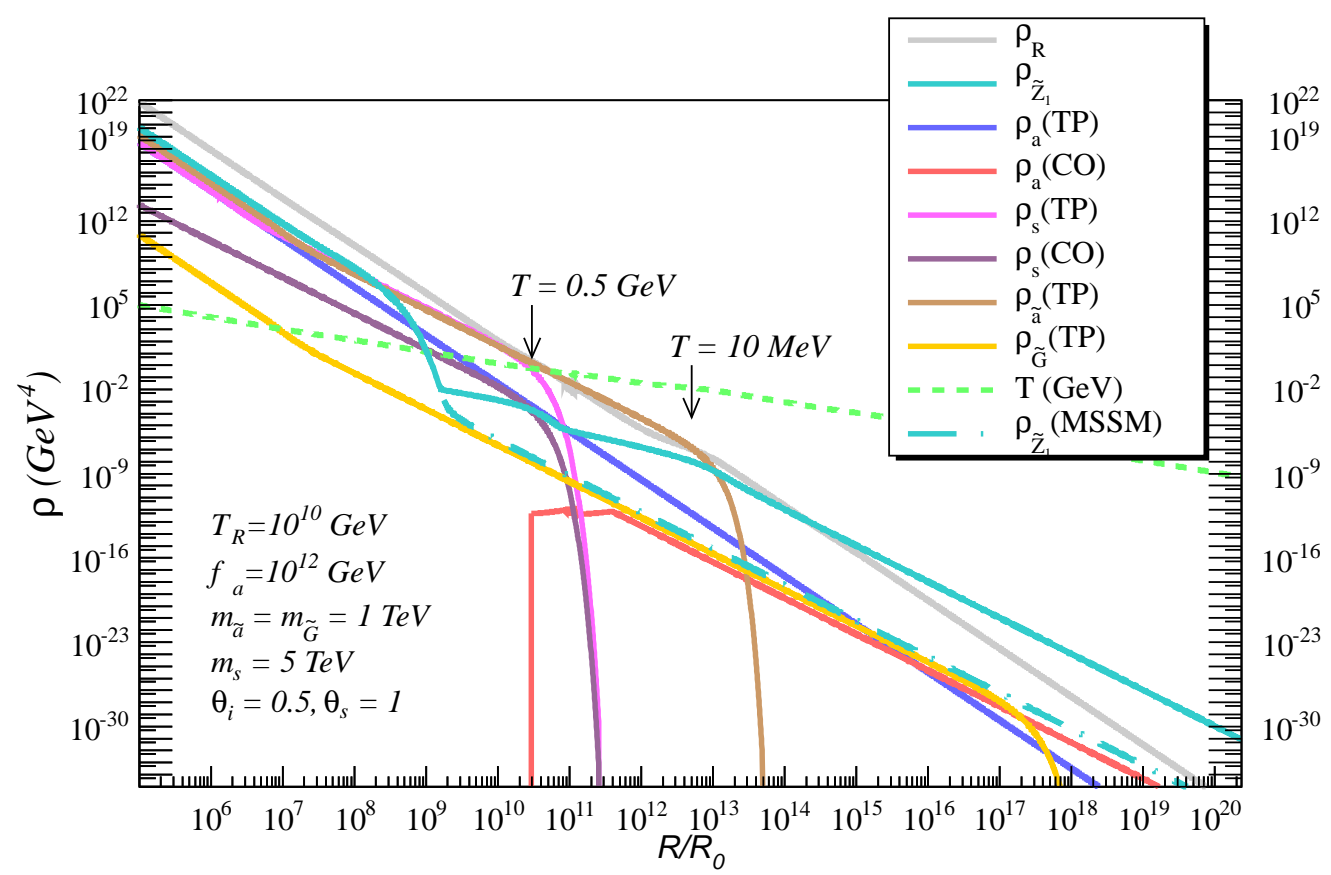

Figure 1: Evolution of radiation, neutralino, axion, saxion, axino and gravitino energy densities versus scale factor $R$. We adopt an mSUGRA SUSY model with parameters $\left(m_{0}, m_{1 / 2}, A_{0}, \tan \beta, \operatorname{sign}(\mu)\right)=(400 \mathrm{GeV}, 400 \mathrm{GeV}, 0,10,+)$. We also take $m_{\widetilde{G}}=1 \mathrm{TeV}$ and $T_{R}=10^{10} \mathrm{GeV}$ and PQ parameters $m_{\tilde{a}}=1 \mathrm{TeV}, m_{s}=5 \mathrm{TeV}, \theta_{i}=0.5, \theta_{s}=1$ with $f_{a}=10^{12}$ $\mathrm{GeV}$.

the universe moves from radiation domination to matter (neutralino) domination, while at even lower temperatures, the gravitinos decay away. In this case, the final neutralino abundance is $\Omega_{\widetilde{Z}_{1}} h^{2} \sim 90017$ - far beyond its standard value. This is mainly due to its abundance being augmented by thermal axino and saxion production and cascade decay to neutralinos. In the standard axion cosmology, the axion abundance would have been $\Omega_{a}^{s t d} h^{2} \sim 0.06[39$. In the case illustrated here, entropy injection from saxion, axino and gravitino decays has diluted its abundance to just $\Omega_{a} h^{2} \sim 0.004$.

As an example of the relevance of using the full set of Boltzmann equations instead of the semi-analytical approach of Refs. [24] and [26], we compare in Fig. 22 the neutralino and axion relic densities as a function of the axino mass using the Boltzmann equation formalism and the semi-analytical approach. The other PQMSSM parameters are the same as used in Fig. 1, but to compare with the semi-analytical results of Refs. 24] and 26] we neglect the saxion component. For these choices of PQ parameters and for $m_{\tilde{a}} \lesssim 50 \mathrm{TeV}$, the axino decays after neutralino freeze-out (as seen on Fig. 1), significantly enhancing its final relic abundance. Furthermore, the axino decay injects entropy, diluting the axion abundance. As we can see from Fig. 2, the axion relic density obtained using the analytical expressions derived in Ref. [26] agree extremely well with the solution of the Boltzmann equations. On the other hand, the analytic neutralino abundance disagrees with the Boltzmann solution by almost an order of magnitude for $m_{\tilde{a}} \lesssim 50 \mathrm{TeV}$. The primary reason for this is the fact 


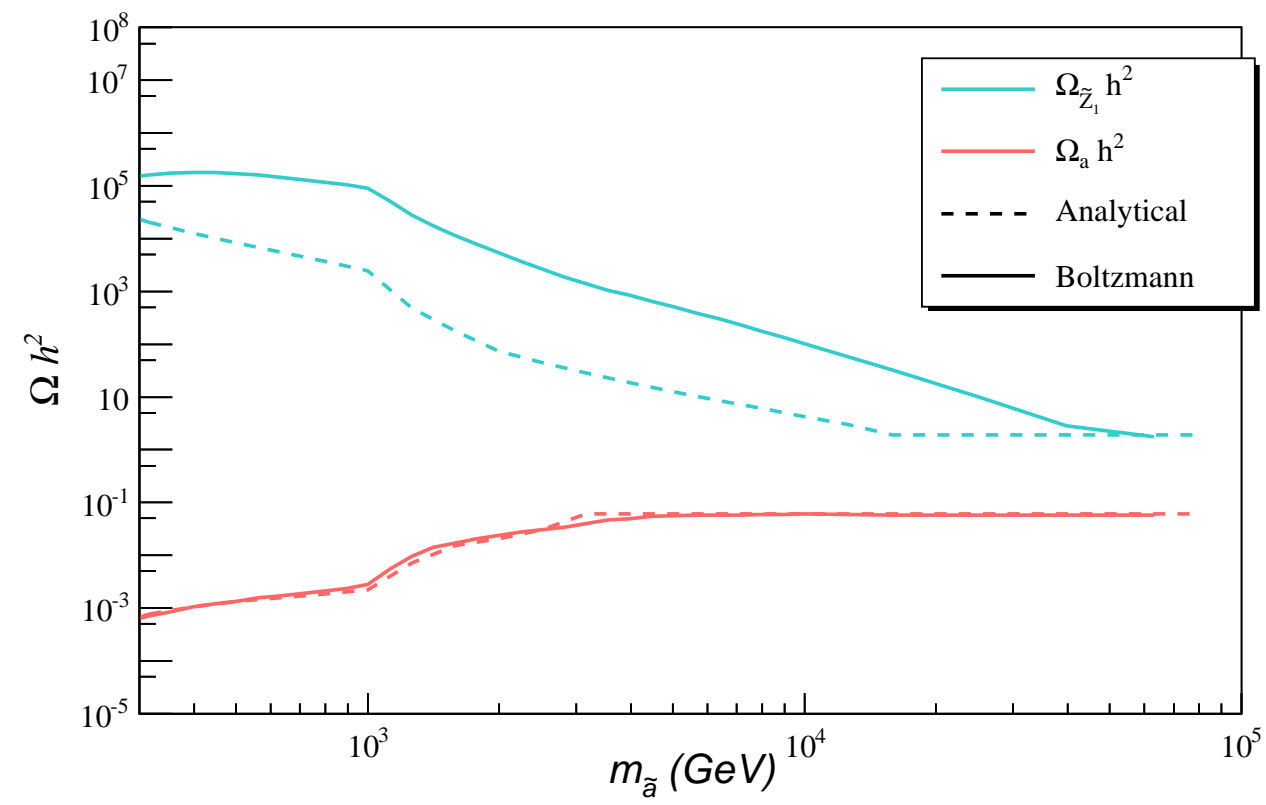

Figure 2: Neutralino and axion relic densities as a function of the axino mass for $\theta_{i}=0.5$, $T_{R}=10^{10} \mathrm{GeV}, f_{a}=10^{12} \mathrm{GeV}$ and the mSUGRA point $\left(m_{0}, m_{1 / 2}, A_{0}, \tan \beta, \operatorname{sign}(\mu)\right)=$ $(400 \mathrm{GeV}, 400 \mathrm{GeV}, 0,10,+)$. The solid lines correspond to the solution of the Boltzmann equations while the dashed lines correspond to the results obtained using the analytical expressions derived in Ref. [26].

that, for this mSUGRA point, the neutralino is bino-like and $\langle\sigma v\rangle_{\widetilde{Z}_{1}}$ is no longer constant, but strongly depends on the temperature. This dependence has not been included in the semi-analytical approach. Also, the sharp transition seen in the semi-analytical result at $m_{\tilde{a}} \simeq 18 \mathrm{TeV}$, where $T_{D}^{\tilde{a}}$ becomes bigger than $T_{f r}$, is artificially introduced by the sudden decay approximation. As shown by the Boltzmann solution, the enhancement of the neutralino relic abundance smoothly decreases, going up to $m_{\tilde{a}} \simeq 50 \mathrm{TeV}$.

\section{Neutralino abundance in the PQMSSM}

\subsection{Neutralino Abundance in several PQMSSM models}

In this section, we adopt four SUSY benchmark models listed in Table 11. The first two points, labeled BM1 and BM2, are generic mSUGRA points with a bino-like $\widetilde{Z}_{1}$ which give rise as expected to an apparent excess of CDM. For BM1, with $\left(m_{0}, m_{1 / 2}, A_{0}, \tan \beta\right.$, $\operatorname{sign}(\mu))=(400 \mathrm{GeV}, 400 \mathrm{GeV}, 0,10,+)$ we have $m_{\widetilde{Z}_{1}}=162.8 \mathrm{GeV}$ with a standard abundance $\Omega_{\widetilde{Z}_{1}}^{\text {std }} h^{2}=1.9$, while the second point $(\mathrm{BM} 2)$ has $\left(m_{0}, m_{1 / 2}, A_{0}, \tan \beta, \operatorname{sign}(\mu)\right)=$ $(3000 \mathrm{GeV}, 1000 \mathrm{GeV}, 0,10,+)$ with $m_{\widetilde{Z}_{1}}=436.3 \mathrm{GeV}$ and $\Omega_{\widetilde{Z}_{1}}^{s t d} h^{2}=49.6$. The next point, BM3, is a mSUGRA point with an apparent underabundance of neutralino dark matter, with $m_{\widetilde{Z}_{1}}=163.8 \mathrm{GeV}, m_{A}=367.5 \mathrm{GeV}$, lying in the $A$-funnel region 40], so 


\begin{tabular}{lcccc}
\hline & BM1 & BM2 & BM3 & BM4 \\
\hline$m_{0}$ & 400 & 3000 & 400 & 0 \\
$m_{1 / 2}$ & 400 & 1000 & 400 & AMSB \\
$m_{3 / 2}$ & $10^{3}$ & $10^{3}$ & $10^{3}$ & $5 \times 10^{4}$ \\
$\tan \beta$ & 10 & 10 & 55 & 10 \\
\hline$m_{\widetilde{Z}_{1}}$ & 162.9 & 436.3 & 163.8 & 142.1 \\
\hline$\Omega_{\widetilde{Z}_{1}}^{s t d} h^{2}$ & 1.9 & 49.6 & 0.019 & 0.0016 \\
$\sigma^{S I}\left(\widetilde{Z}_{1} p\right) \mathrm{pb}$ & $8.1 \times 10^{-10}$ & $1.1 \times 10^{-10}$ & $2.1 \times 10^{-8}$ & $4.3 \times 10^{-9}$ \\
\hline
\end{tabular}

Table 1: Masses and parameters in $\mathrm{GeV}$ units for several benchmark points computed with ISAJET 7.81 using $A_{0}=0$ and $m_{t}=173.3 \mathrm{GeV}$.

$\Omega_{\widetilde{Z}_{1}}^{s t d} h^{2}=0.019$. For all cases, we take $m_{\widetilde{G}}=1 \mathrm{TeV}$, but now will vary the PQ parameters and $T_{R}$, in order to see if the relic density of mixed $a \widetilde{Z}_{1}$ CDM can lie in the WMAP-allowed region. The last point is taken from the gaugino AMSB model 41, 42] and has a wino-like neutralino with $\Omega_{\widetilde{Z}_{1}}^{s t d} h^{2}=0.0016$, but with $m_{3 / 2} \equiv m_{\widetilde{G}}=50 \mathrm{TeV}$.

In order to keep our results as general as possible, we will not assume particular PQ parameters, but instead we scan over the following parameter values:

$$
\begin{aligned}
10^{9} \mathrm{GeV} & <f_{a}<10^{16} \mathrm{GeV}, \\
500 \mathrm{GeV} & <m_{\tilde{a}}<10^{4} \mathrm{GeV}, \\
10^{3} \mathrm{GeV} & <m_{s}<10^{5} \mathrm{GeV}, \\
0.1 & <\theta_{s}<10, \\
10^{5} \mathrm{GeV} & <T_{R}<10^{12} \mathrm{GeV} .
\end{aligned}
$$

Since we will be mostly concerned with the neutralino relic abundance, we leave the axion mis-alignment angle $\theta_{i}$ undetermined for now.

\subsection{Benchmark BM1}

Our results are shown as the resultant relic density of neutralinos $\Omega_{\widetilde{Z}_{1}} h^{2}$ in the PQMSSM, where we plot each model versus $f_{a}$ in Fig. 3. The blue points are labeled as BBN-allowed, while red points violate the BBN bounds as described in Sec. 2.2.

From Fig. 3, we see that at low values of PQ breaking scale $f_{a} \sim 10^{9}-10^{11} \mathrm{GeV}$, the value of $\Omega_{\widetilde{Z}_{1}} h^{2}$ is always bounded from below by its standard value $\Omega_{\widetilde{Z}_{1}}^{s t d} h^{2} \sim 1.9$. Those points with $\Omega_{\widetilde{Z}_{1}} h^{2} \simeq 1.9$ are typically those for which axinos and saxions decay before $T_{f r}$, or those for which axino/saxion production is suppressed by low $T_{R}$ so that axinos/saxions decays do not significantly contribute to $\Omega_{\widetilde{Z}_{1}} h^{2}$.

In Fig. 3, frequently the neutralino abundance is enhanced beyond 1.9, making these points even more excluded. The reason why points only have enhanced relic densities at the lower $f_{a}$ range is because the axino-matter coupling is proportional to $1 / f_{a}$, and so thermal axino production is enhanced compared to higher $f_{a}$ values. In addition, $\tilde{a} \rightarrow \tilde{g} g$ decays may be phase space suppressed, so that axino decay takes place at temperature $T_{D}^{\tilde{a}}<T_{f r}$, thereby augmenting the neutralino abundance. Saxion decay is never phase 


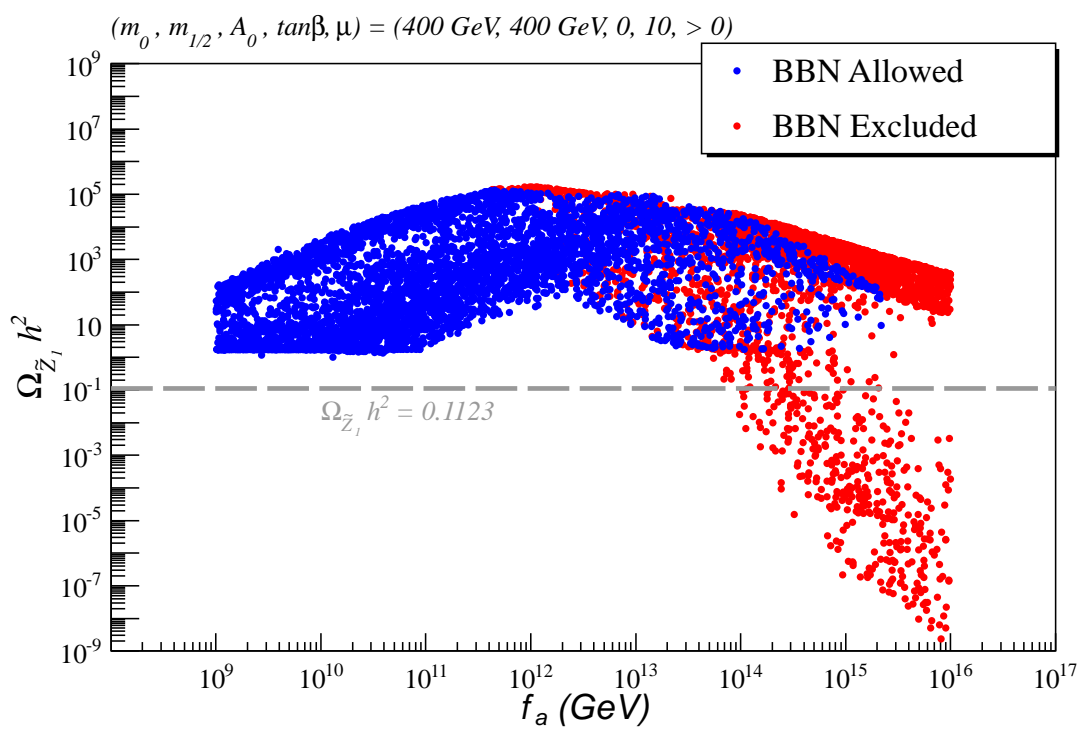

Figure 3: Calculated neutralino relic abundance from mSUGRA model BM1 versus $f_{a}$. We take $m_{\widetilde{G}}=1 \mathrm{TeV}$. The spread in dots is due to a scan over PQ parameters $f_{a}, T_{R}, m_{\tilde{a}}, m_{s}$ and $\theta_{s}$.

space suppressed, since $s \rightarrow g g$ is always possible, so at the lower range of $f_{a}$, saxion decay typically takes place at $T_{D}^{s}>T_{f r}$. For values of $f_{a} \sim 10^{11} \mathrm{GeV}$ and beyond, axinos can no longer decay before neutralino freeze-out, and so the neutralino abundance is always enhanced. The value of $f_{a}$ where the neutralino abundance is always enhanced is somewhat an artifact of our scanning range, since if we allow $m_{\tilde{a}}>10^{4} \mathrm{GeV}$, axinos could become shorter-lived for a higher value of $f_{a}$ since $\Gamma_{\tilde{a}} \sim m_{\tilde{a}}^{3} / f_{a}^{2}$.

At even higher values of $f_{a} \gtrsim 10^{12} \mathrm{GeV}$, axino/saxion thermal production becomes increasingly suppressed, while saxion production via CO becomes enhanced: entropy dilution by saxions starts winning over neutralino production from thermal axino production and decay. Also, both saxion and axino become even longer-lived, so more points become BBN-disallowed. Although the entropy injection from $s \rightarrow g g$ decays grows with $f_{a}$, the BBN-allowed blue points are never pushed below $\Omega_{\widetilde{Z}_{1}} h^{2} \sim 1.9$, since $s \rightarrow \tilde{g} \tilde{g}$ also injects additional neutralinos into the thermal bath. ${ }^{5}$

To understand why the neutralino injection from saxion decays always wins over the entropy dilution of the neutralino abundance, we must look at the neutralino Yield from saxion decays. For simplicity, we will neglect the axino component as well as neutralino re-annihilation at $T_{D}^{s}$ and assume that the PQ parameters are chosen so the universe has a saxion-dominated era, since this is the only scenario with significant entropy injection.

\footnotetext{
${ }^{5}$ We checked the effect of artificially turning off $s \rightarrow \tilde{g} \tilde{g}$ decays in Fig. 3. In this case, at high $f_{a} \gtrsim 10^{14}$ $\mathrm{GeV}$, the enhanced saxion production via COs produces only entropy dilution of the neutralino abundance, and some BBN-allowed points remain with a highly suppressed neutralino abundance at high $f_{a}$. This effect has lead to claims that large $f_{a} \sim M_{G U T}$ values may be allowed in SUSY models due to entropy injection by saxions 43, 44, 45, 46, 47, 48, 49, 50, 51, 52. By properly including $s \rightarrow \tilde{g} \tilde{g}$ decay and the concommitant neutralino re-annihilation at $T_{s}$, the pure entropy injection effect is counter-balanced in this case, and the highly diluted cases become BBN-forbidden.
} 
Under these assumptions, the Yield of neutralinos emitted from saxion decays is simply given by [52]:

$$
Y_{\widetilde{Z}_{1}}=\frac{1}{r} Y_{s} \times 2 B R(s \rightarrow \tilde{g} \tilde{g})
$$

where the factor 2 above takes care of the multiplicity of neutralinos from each saxion decay and $r$ is the entropy injection factor, which can be approximated by

$$
r=\frac{T_{e}}{T_{D}^{s}}
$$

where $T_{D}^{s}$ is the saxion decay temperature and $T_{e}=4 m_{s} Y_{s} / 3$ (see Refs. [52, 53]). Therefore:

$$
Y_{\widetilde{Z}_{1}}=\frac{3}{2} \frac{T_{D}^{s}}{m_{s}} B R(s \rightarrow \tilde{g} \tilde{g}) \Rightarrow \quad \Omega_{\widetilde{Z}_{1}}^{s} h^{2} \simeq 4 \times 10^{8} \mathrm{GeV}^{-1} m_{\widetilde{Z}_{1}} \frac{T_{D}^{s}}{m_{s}} B R(s \rightarrow \tilde{g} \tilde{g})
$$

The above expression shows that the relic density can be suppressed for large $m_{s}$, small $T_{D}^{s}$ and/or small $B R$. However, as seen in Fig. 3, such suppression never seems to drive $\Omega_{\widetilde{Z}_{1}} h^{2}$ below its standard value, except in the BBN excluded region. To see why this happens, using Eq. 3.8 we show in Fig. 国 contours of $\Omega_{\widetilde{Z}_{1}}^{s} h^{2}$ in the $m_{s} v s . f_{a}$ plane for the BM1 point, with $T_{R}=10^{6} \mathrm{GeV}$ and $\theta_{s}=1$. We also show the BBN excluded region $\left(T_{D}^{s}<5 \mathrm{MeV}\right)$ and the region with $T_{D}^{s}>T_{e}(r<1)$, where there is no saxion dominated era and Eq. 3.8 is no longer valid. As we can see, the allowed region (white) can only satisfy the WMAP constraints at very large $m_{s}$ and $f_{a}$ values. The main reason for the low $\Omega_{\widetilde{Z}_{1}} h^{2}$ values obtained in this region is due to the suppression of $B R(s \rightarrow \tilde{g} \tilde{g})$. This can be seen in Fig. 5. where we show the branching ratio as a function of $m_{s}$ for the same benchmark point. We can see that- for the region where the $s \rightarrow \tilde{g} \tilde{g}$ decay mode is closed- the saxion lifetime falls into the BBN-forbidden zone. This can also be seen in Fig. 3, where all the low $\Omega_{\widetilde{Z}_{1}} h^{2}$ points at large $f_{a}$ are BBN excluded.

As seen from the above results, the neutralino relic abundance can indeed be diluted by including the saxion field, but only at the expense of going to extremely high $m_{s}$ and $f_{a}$ values. However, since $m_{s}$ is expected to be of order the soft SUSY masses (or $\sim m_{\widetilde{G}}$ in e.g. AMSB models), we consider such high values extremely unnatural. Furthermore, the PQMSSM is most likely not the correct effective theory at $f_{a}>10^{16}-10^{19} \mathrm{GeV}$, where we expect a Grand Unified theory and/or large supergravity corrections. Nonetheless, to confirm the approximate results obtained from Eq. 3.8, we extend our previous scan over to

$$
f_{a} \in\left[10^{15}, 10^{22}\right] \mathrm{GeV}, \quad m_{s} \in\left[10^{4}, 10^{9}\right] \mathrm{GeV}
$$

and use the full set of Boltzmann equations to compute the neutralino relic abundance. The results are shown in Fig. 6, where we plot in the $m_{s} v s . f_{a}$ plane all solutions satisfying $\Omega_{\widetilde{Z}_{1}} h^{2}<0.11$. As we can see, the numerical results agree very well with the analytical results in Fig. 6 . The only discrepancy is in the region near $T_{e}=T_{D}^{s}$, which does not present viable solutions in the scan. This is simply due to the fact that in our estimate of Eq. 3.8, we neglected the neutralino freeze-out component, which becomes dominant when $r \simeq 1$ or $T_{e} \simeq T_{D}^{s}$, increasing the value of $\Omega_{\widetilde{Z}_{1}} h^{2}$ in this region. 


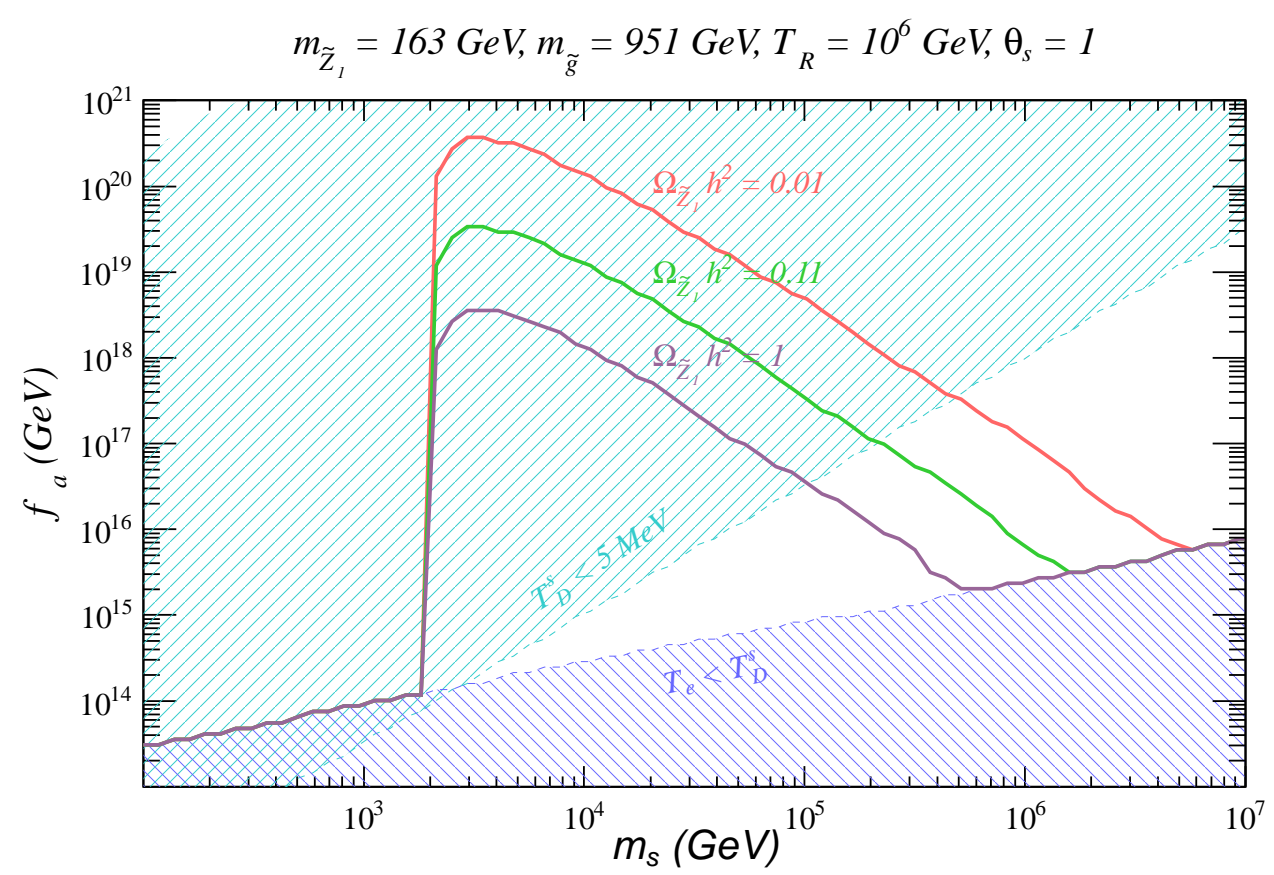

Figure 4: Regions in the $m_{s} v s . f_{a}$ plane where $T_{D}^{s}<5 \mathrm{MeV}$ and $T_{D}^{s}>T_{e}$. We also show contours of constant $\Omega_{\widetilde{Z}_{1}}^{s} h^{2}$ as estimated using Eq. 3.8.

From the results presented above, we see that for reasonable values of $f_{a}, m_{s}$ and $\theta_{s}$, the result illustrated for point BM1 in Fig. 3 seems to generalize to all SUSY model points with a standard neutralino overabundance: SUSY models with a standard overabundance of neutralino dark matter are still at least as excluded when augmented by the $P Q$ mechanism.

In Fig. 7, we show the evolution of various energy densities versus the scale factor for a large $f_{a}$ value. In this case, we see that the universe is radiation-dominated out to $R / R_{0} \sim 10^{8}$, whereupon it becomes saxion dominated. If only $s \rightarrow g g$ is considered, the saxion entropy injection would cause a large dilution of neutralinos. But by including $s \rightarrow \tilde{g} \tilde{g}$ decays, we see the neutralino enhancement during $10^{7} \lesssim R / R_{0} \lesssim 10^{9}$. We also show by the dash-dotted line the expected neutralino energy density by neglecting $s \rightarrow \tilde{g} \tilde{g}$ decays: in this case, the neutralino abundance is highly suppressed compared to the case where $s \rightarrow \tilde{g} \tilde{g}$ is accounted for.

\subsection{Benchmark BM2}

To emphasize some of the generality of our previous results, we show a further point with a standard overabundance of neutralinos in Fig. 8, with $\left(m_{0}, m_{1 / 2}, A_{0}, \tan \beta, \operatorname{sign}(\mu)\right)=$ $(3000 \mathrm{GeV}, 1000 \mathrm{GeV}, 0,10,+)$, for which $\Omega_{\widetilde{Z}_{1}}^{s t d} h^{2} \sim 50$. By scanning over PQ parameters, again we find that for low $f_{a}, \Omega_{\widetilde{Z}_{1}} h^{2}$ either remains at its standard value (if axinos/saxions decay before freeze-out), or are enhanced (if axinos/saxions decay after freeze-out). At high $f_{a}$, entropy dilution from CO-produced saxions again can suppress $\Omega_{\widetilde{Z}_{1}} h^{2}$, but the 


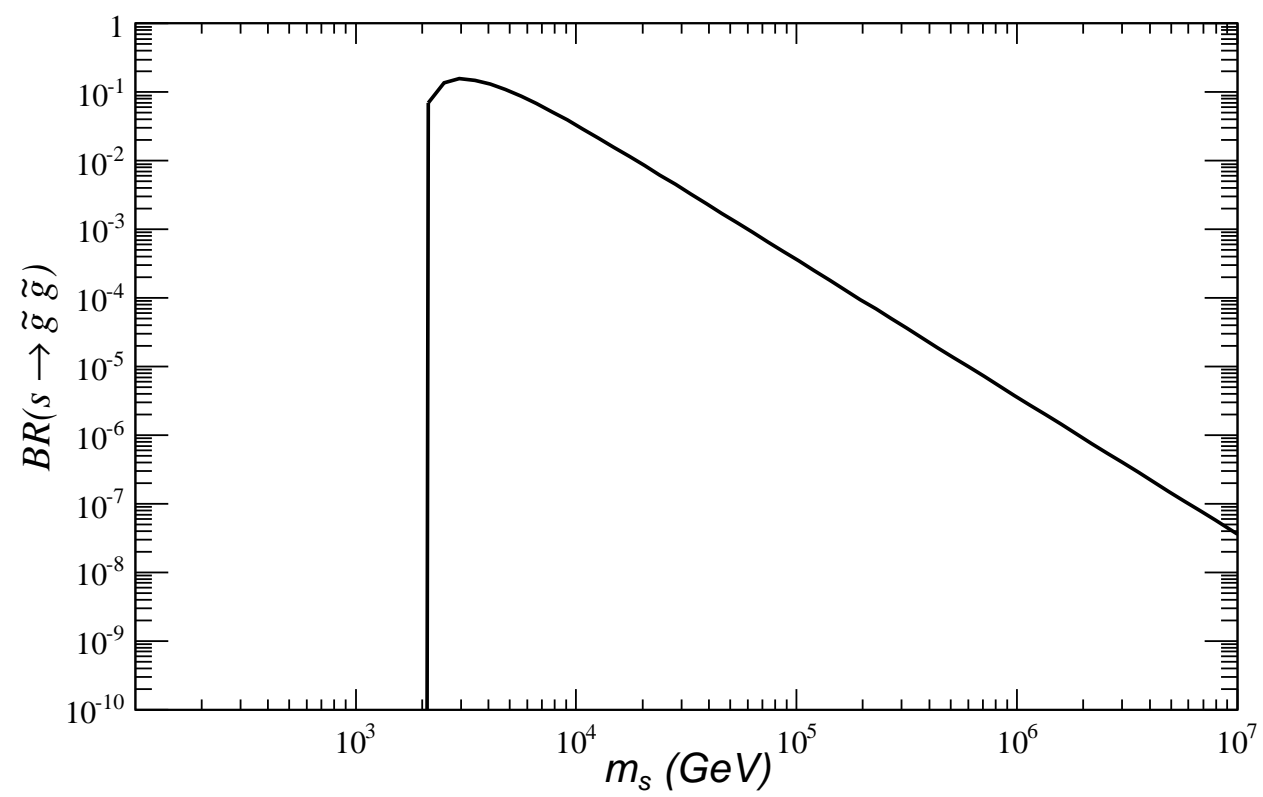

Figure 5: Branching ratio of saxion decays into gluino pairs as a function of $m_{s}$, for $m_{\tilde{g}}=951$ $\mathrm{GeV}$.

suppression is counterbalanced by $s \rightarrow \tilde{g} \tilde{g}$ decays: only BBN-forbidden points where $m_{s}$ is so light that $s \rightarrow \tilde{g} \tilde{g}$ is kinematically closed yield points with $\Omega_{\widetilde{Z}_{1}} h^{2}<0.11$.

\subsection{Benchmark BM3: A-resonance region}

In Fig. 9, we show the neutralino abundance in the case of an mSUGRA point lying in the $A$-resonance annihilation region[40] where $2 m_{\widetilde{Z}_{1}} \sim m_{A}$. We adopt mSUGRA parameters $\left(m_{0}, m_{1 / 2}, A_{0}, \tan \beta, \operatorname{sign}(\mu)\right)=(400 \mathrm{GeV}, 400 \mathrm{GeV}, 0,55,+)$, for which $\Omega_{\widetilde{Z}_{1}}^{s t d} h^{2} \sim 0.02$, i.e. a standard underabundance. ${ }^{6}$ In this case, a scan over PQ parameters yields many points at low $f_{a}$ with $\Omega_{\widetilde{Z}_{1}} h^{2} \sim 0.02-10$. Thus, the standard neutralino underabundance may be enhanced up to the WMAP-allowed value, or even beyond. As we push to higher $f_{a}$ values, the axino becomes so long-lived that it only decays after neutralino freeze-out, and hence the neutralino abundance is always enhanced. Above $f_{a} \sim 10^{12} \mathrm{GeV}$, the neutralino abundance is enhanced into the WMAP-forbidden region, with $\Omega_{\widetilde{Z}_{1}} h^{2}$ always larger than 0.11 . As we push even higher in $f_{a}$, then axino production is suppressed, but CO-production of saxions becomes large. Entropy dilution turns the range of $\Omega_{\widetilde{Z}_{1}} h^{2}$ back down again, and at $f_{a} \sim 10^{14} \mathrm{GeV}$, some BBN-allowed points again reach $\Omega_{\widetilde{Z}_{1}} h^{2} \sim 0.11$. In this case, rather large $f_{a}$ values approaching $M_{G U T}$ are allowed.

For the points with $\Omega_{\widetilde{Z}_{1}} h^{2}<0.11$, the remaining dark matter abundance can be accommodated by axions via a suitable adjustment of the initial axion mis-alignment angle

\footnotetext{
${ }^{6}$ We have also scanned a benchmark point in the hyperbolic branch/focus point region[55] of mSUGRA, again with a standard underabundance of neutralino dark matter. The $\Omega_{\widetilde{Z}_{1}} h^{2}$ vs. $f_{a}$ results look qualitatively much like Fig. 9. We do not present these results here in the interests of brevity.
} 


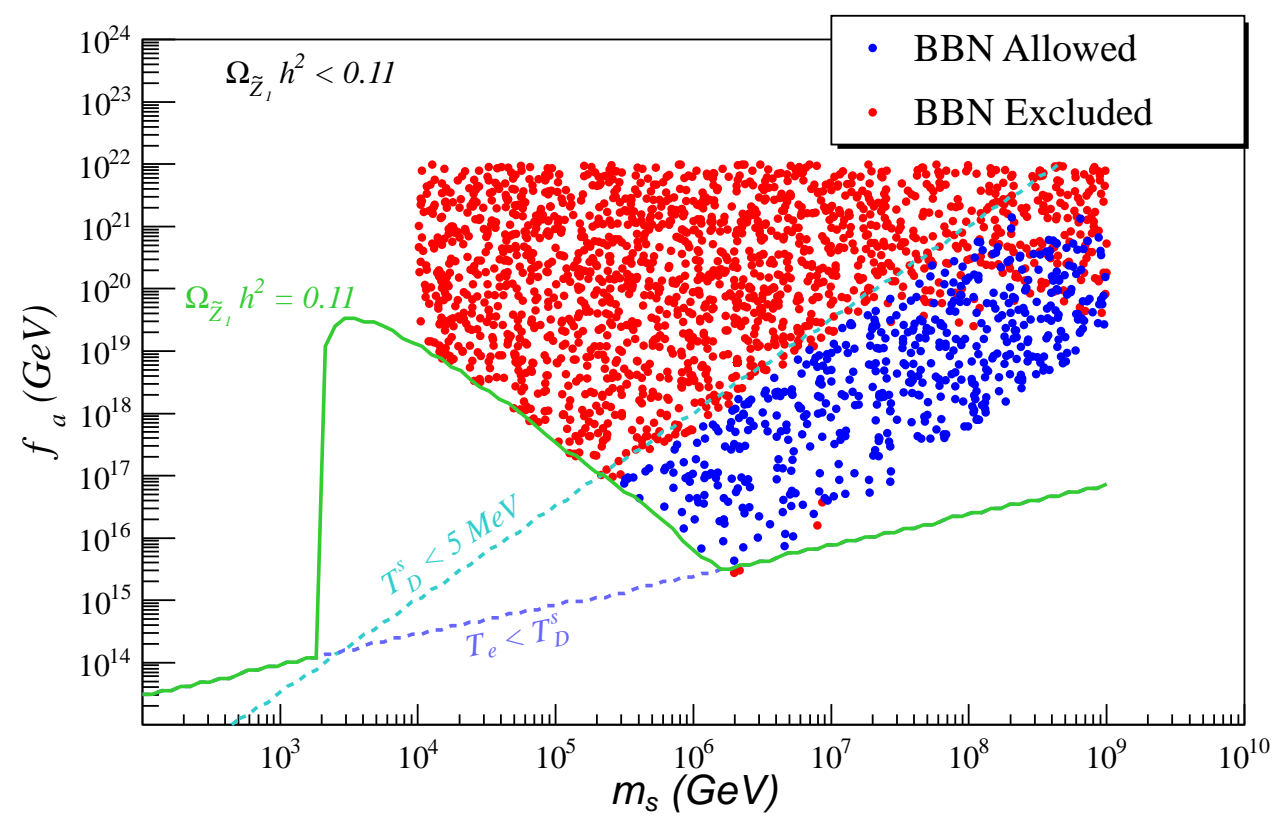

Figure 6: Points with $\Omega_{\widetilde{Z}_{1}} h^{2}<0.11$ obtained through the random scan described in the text. For comparison, we also show the curves for $T_{D}^{s}<5 \mathrm{MeV}$ and $T_{D}^{s}>T_{e}$ and $\Omega_{\widetilde{Z}_{1}}^{s} h^{2}=0.11$ obtained from Eq. 3.8.

$\theta_{i}$. In Fig. 10, we show the required value of $\theta_{i}$ needed to enforce $\Omega_{\widetilde{Z}_{1}} h^{2}+\Omega_{a} h^{2}=0.11$. At low $f_{a}$, the points satisfying $\Omega_{\widetilde{Z}_{1}} h^{2}<0.11$ have axinos and saxions decaying before the neutralino freezes out and consequently before axions start to oscillate. Hence the axion relic density is not affected by the entropy injection of axinos/saxions and is given by the standard expression 39]:

$$
\Omega_{a} h^{2} \simeq 0.23 \theta_{i}^{2}\left(\frac{f_{a}}{10^{12} \mathrm{GeV}}\right)^{7 / 6}
$$

From the above equation, we see that as $f_{a}$ increases, $\theta_{i}$ must decrease in order to maintain $\Omega_{\widetilde{Z}_{1}} h^{2}+\Omega_{a} h^{2}=0.11$. This behavior is clearly seen in Fig. 10 for $f_{a}<10^{12} \mathrm{GeV}$. Once $f_{a}$ becomes sufficiently large so axinos and saxions start to decay after the axion starts to oscillate, the entropy injected from saxions and axinos considerably dilute the axion relic density, thus allowing for larger $\theta_{i}$ values. However, as seen in Fig. 10, this only happens for the BBN-forbidden solutions at $f_{a} \gtrsim 10^{14} \mathrm{GeV}$. The only BBN-allowed points at large $f_{a}$ with $\Omega_{\widetilde{Z}_{1}} h^{2}<0.11$ are the ones where the saxion production is either suppressed or where it decays before neutralino freeze-out. In this case there is no significant entropy injection and the axion relic density is once again given by Eq. 3.10. Thus, extremely small values of $\theta_{i}$ are required in order to suppress the axion relic density at large $f_{a}$, as seen in Fig. 10. Therefore these points tend to have neutralino domination of the dark matter density, rather than axion domination. For these points, the large neutralino haloannihilation rates, enhanced by the $A$-resonance, may lead to visible production rates of 


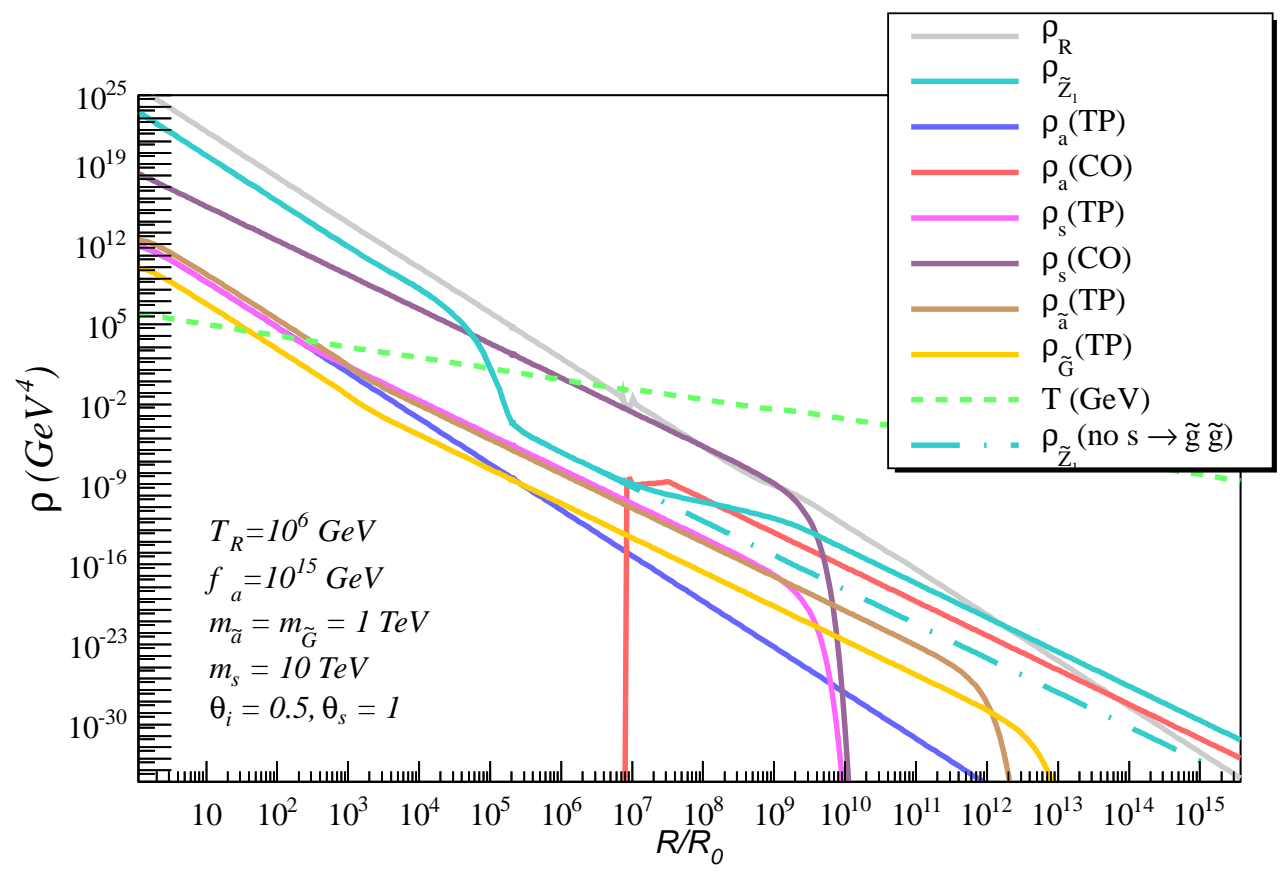

Figure 7: Evolution of radiation, neutralino, axion, saxion, axino and gravitino energy densities versus scale factor $R$ starting at $T=T_{R}$. We adopt an mSUGRA SUSY model with parameters $\left(m_{0}, m_{1 / 2}, A_{0}, \tan \beta, \operatorname{sign}(\mu)\right)=(400 \mathrm{GeV}, 400 \mathrm{GeV}, 0,10,+)$. The PQ parameters are listed on the plot.

$\gamma \mathrm{s}, e^{+} \mathrm{s}$ and $\bar{p} \mathrm{~s}$ in cosmic ray detectors [56], while corresponding direct neutralino detection rates may remain low.

\subsection{Benchmark BM4: AMSB with wino-like neutralino}

In Fig. 11, we plot $\Omega_{\widetilde{Z}_{1}} h^{2}$ for an anomaly-mediated SUSY breaking model (AMSB) with a wino-like neutralino 41. We choose the gaugino-AMSB model with $m_{0} \sim A_{0} \sim 0$, since this model avoids tachyonic sleptons without introduction of an additional scalar mass parameter 42]. Model parameters are $\left(m_{3 / 2}, \tan \beta, \operatorname{sign}(\mu)\right)=(50 \mathrm{TeV}, 10,+)$, with a standard abundance $\Omega_{\widetilde{Z}_{1}}^{\text {std }} h^{2} \simeq 0.0016$, far below the measured value. From the figure, we see that for $f_{a} \sim 10^{9}-10^{15} \mathrm{GeV}$, the neutralino abundance can be enhanced and brought into accord with measured values. For low $f_{a}$, axino production and decay augments the abundance, while for high $f_{a}$, saxion production and decay both augments and dilutes the abundance. In this case, as with BM3, the standard underabundance of DM can be augmented and brought into accord with cosmological measurements. Unlike BM3, there exists no intermediate range of $f_{a}$ which is always excluded by the production of too much neutralino DM. The PQ scale can be as large as $f_{a} \sim 10^{15} \mathrm{GeV}$, in accord with expectations from string theory.

Originally, Moroi and Randall had proposed augmenting the relic wino abundance from AMSB via moduli production and decay [57, 58, 59]. Here, we see that an alternative 


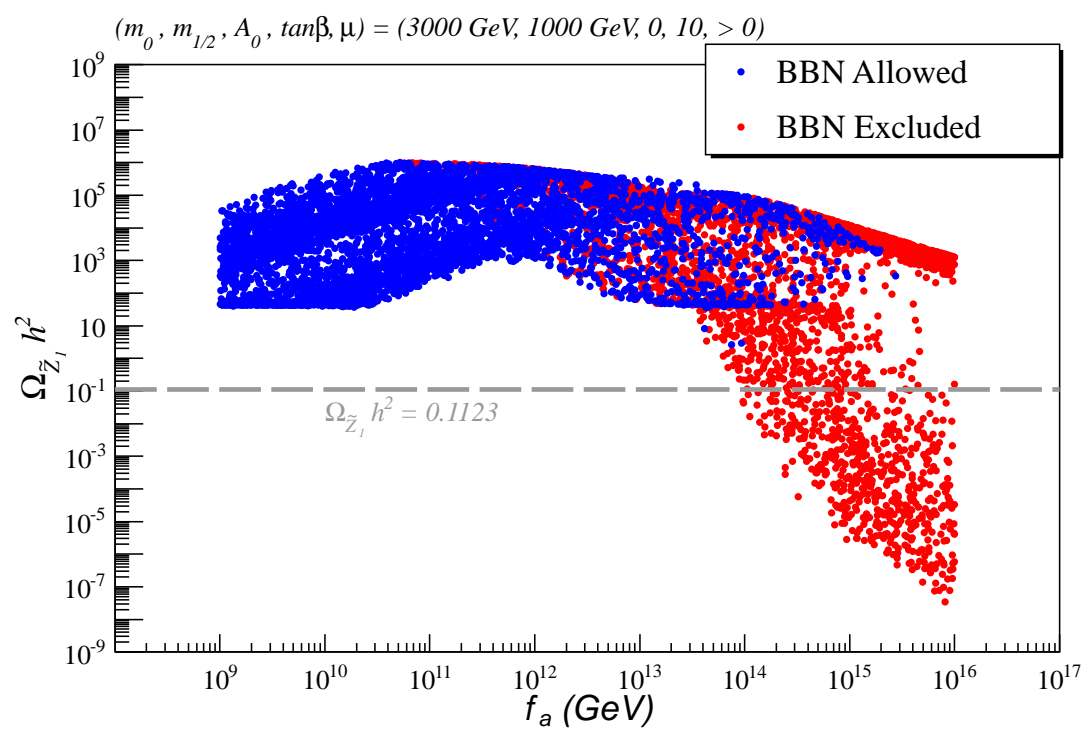

Figure 8: Calculated neutralino relic abundance versus $f_{a}$ from mSUGRA SUSY model BM2. The spread in dots is due to a scan over PQ parameters $f_{a}, T_{R}, m_{\tilde{a}}, m_{s}, \theta_{s}$.

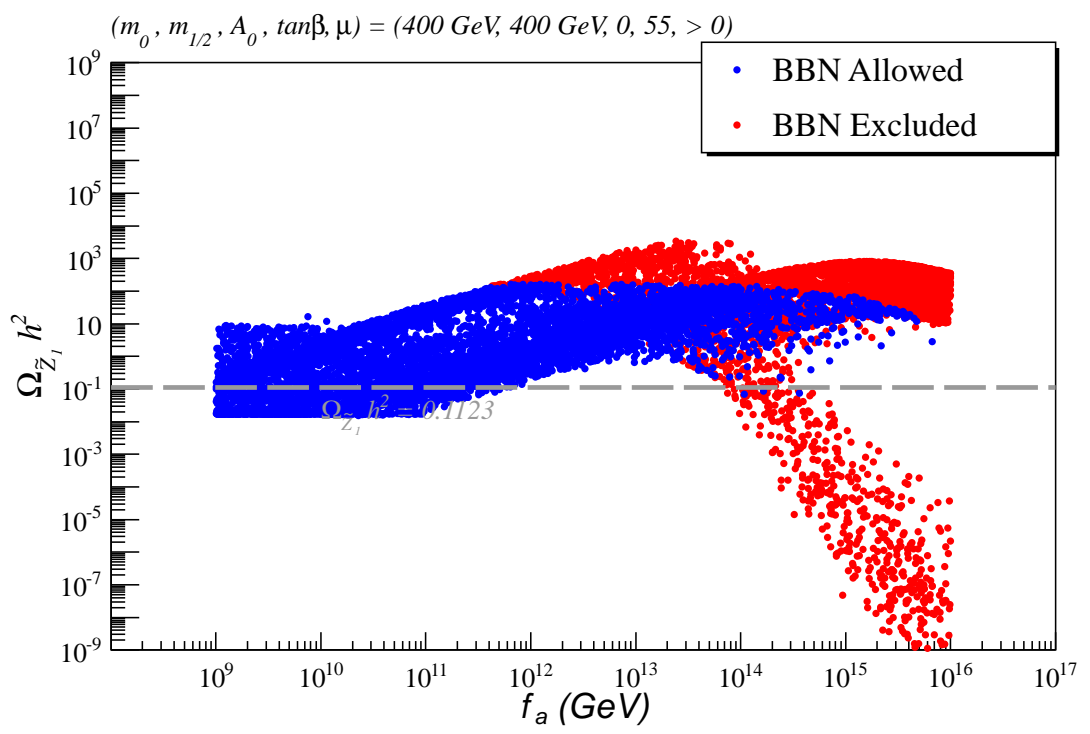

Figure 9: Calculated neutralino relic abundance versus $f_{a}$ from mSUGRA SUSY model BM3. The spread in dots is due to a scan over PQ parameters $f_{a}, T_{R}, m_{\tilde{a}}, m_{s}, \theta_{s}$.

mechanism introducing the several PQMSSM fields can also do the job. Direct and indirect detection rates for wino-like neutralinos have been presented in Ref. [60].

\section{The case of very large $\theta_{s}$ and $m_{s}<2 m_{\tilde{g}}$}

From the results presented in Secs. 3.2 3.5, it seems difficult to suppress the neutralino 


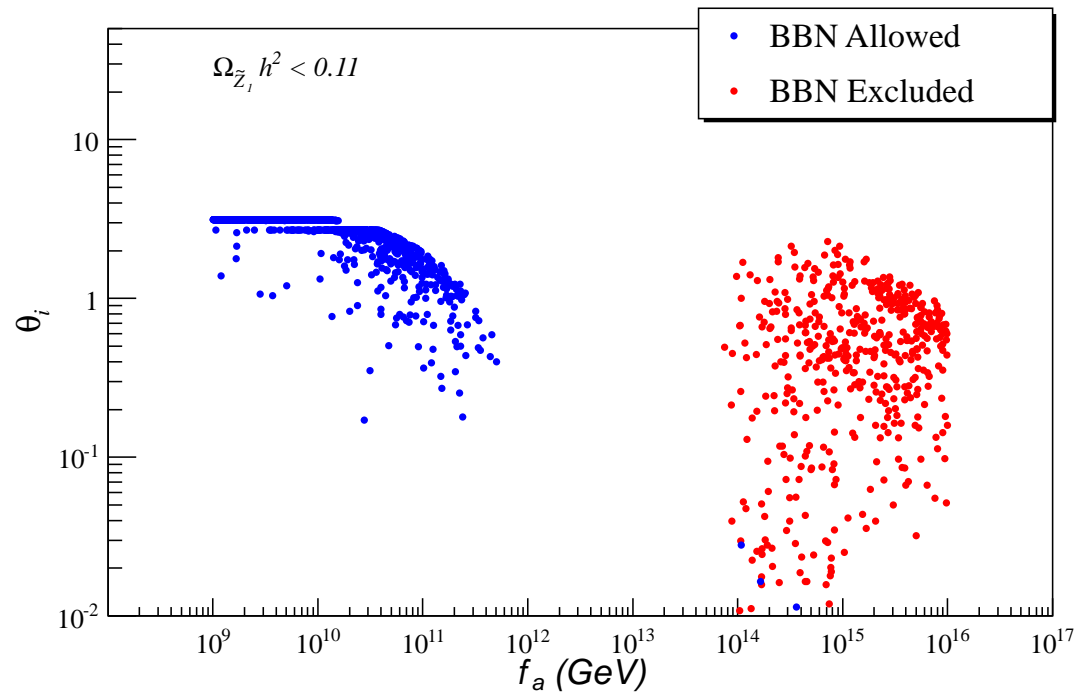

Figure 10: Values of the axion mis-alignment angle $\theta_{i}$ for the points in Fig. 9 with $\Omega_{\widetilde{Z}_{1}} h^{2}<0.11$. The parameter $\theta_{i}$ is chosen such as $\Omega_{\widetilde{Z}_{1}} h^{2}+\Omega_{a} h^{2}=0.11$.

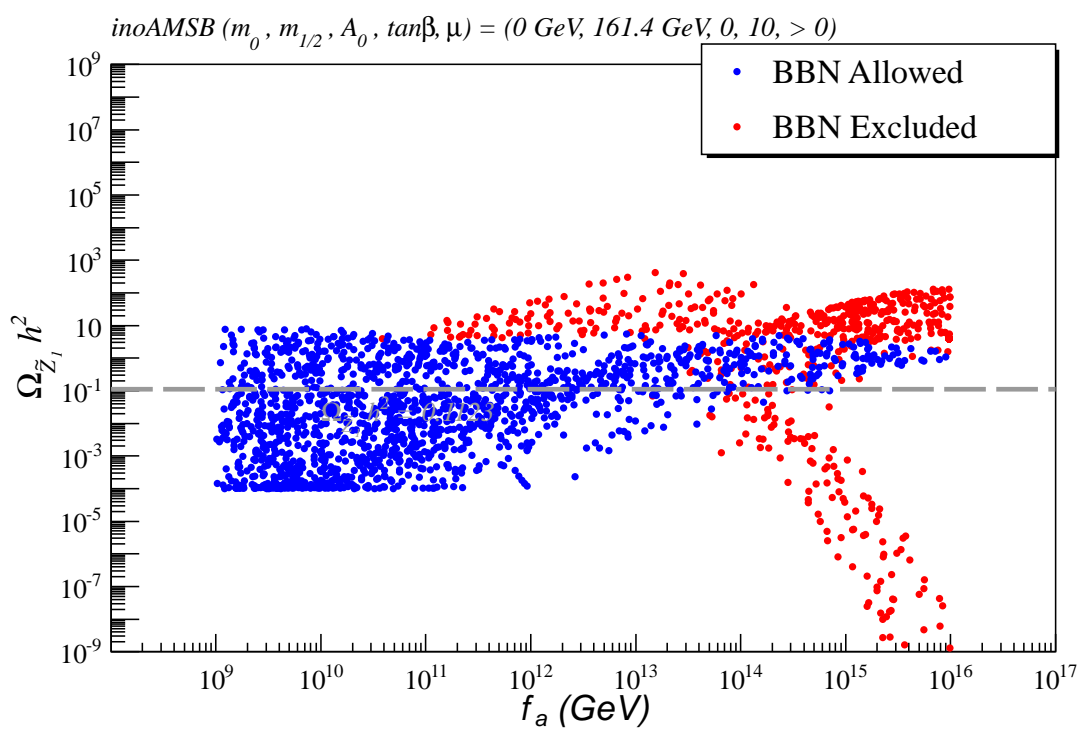

Figure 11: Calculated neutralino relic abundance versus $f_{a}$ from inoAMSB model BM4. The spread in dots is due to a scan over PQ parameters $f_{a}, T_{R}, m_{\tilde{a}}, m_{s}, \theta_{s}$.

CDM abundance below the standard neutralino abundance. This conclusion relies on the fact that the $(\mathrm{CO})$ saxion production and decay are correlated through the value of the PQ scale, since the saxion field strenght $\left(s(x)=\theta_{s} f_{a}\right)$ - which sets the amplitude of the coherent oscillations- is assumed to be of order $f_{a}\left(\theta_{s} \sim \mathcal{O}(0.1-10)\right)$. Hence large $(\mathrm{CO})$ saxion production only happens at large $f_{a}$ values and leads to late decaying saxions, usually 
violating the BBN bounds. As also discussed above, the BBN bounds can be avoided if saxions have masses in the multi-TeV range, but then the $s \rightarrow \tilde{g} \tilde{g}$ decay is kinematically allowed and the injection of neutralinos enhances the CDM abundance. However, if the saxion field strength $(s(x))$ is not set by the $\mathrm{PQ}$ breaking scale, but by a much larger scale, such as the reduced Planck mass (as suggested in some models [54), it is possible to envision a large production of coherent oscillating saxions even at small $f_{a}$ values. In this scenario, small $f_{a}$ easily satisfies the BBN bounds, allowing for sub-TeV saxion masses, such as $m_{s}<2 m_{\tilde{g}}$. Thus, assuming $s(x) \gg f_{a}\left(\theta_{s} \gg 1\right)$, it is possible to have large saxion production via coherent oscillations, small $f_{a}$ values and small saxion masses without violating the BBN bounds. In this case, if $m_{s}<2 m_{\tilde{g}}$, saxion decay leads to large entropy production, but does not inject neutralinos.

To illustrate the large $\theta_{s}(\gg 1)$ scenario, in Fig. 12 we fix the initial saxion field strength to $s(x)=\theta_{s} f_{a}=5 \times 10^{17} \mathrm{GeV}$, but allow $f_{a}$ to vary and compute the neutralino and axion CDM abundances assuming $m_{\widetilde{G}}=m_{s}=m_{\tilde{a}}=1 \mathrm{TeV}, T_{R}=10^{6} \mathrm{GeV}, \theta_{i}=0.5$ and the BM1 benchmark point. In this case, $m_{s}<2 m_{\tilde{g}}$ so that if saxions can dominate the energy density of the universe, they only lead to entropy dilution, and not CDM production. From the plot, we see that for low $f_{a}$ the neutralino abundance is enhanced due to large thermal production of axinos and their decay to neutralinos. As $f_{a}$ increases, thermal production of axinos and saxions becomes suppressed, while the saxion decay temperature decreases, leading to increased entropy dilution of the neutralino abundance. At $f_{a} \sim 10^{12} \mathrm{GeV}, \Omega_{\widetilde{Z}_{1}} h^{2}$ drops below 0.1 , and the BM1 point becomes allowed in the PQMSSM. Meanwhile, the axion abundance will also suffer entropy dilution as $f_{a}$ increases, but this is counterbalanced by an increasing axion field strength, which leads to greater axion production via COs: the net result is an almost flat value of $\Omega_{a} h^{2}$ as $f_{a}$ varies. Once $f_{a}$ increases past $\sim 10^{13} \mathrm{GeV}$, the saxion becomes sufficiently long-lived that the model begins to violate $\mathrm{BBN}$ bounds. While this scenario does provide a strong dilution of dark matter relics, we note here that the values of $\theta_{s}$ needed are in the range $\theta_{s} \sim 10^{5}-10^{6}$ so that the saxion field strength is far beyond the value of $f_{a}$ and must be given by another physics scale.

\section{Conclusions}

In this paper we have presented the results of a calculation of mixed axion/neutralino CDM abundance using a set of eight coupled Boltzmann equations. The calculation improves upon previous results in several respects: 1. it allows for non-constant values of $\langle\sigma v\rangle$, as occurs for bino-like neutralinos, where $s$-wave annihilation is suppressed, 2 . it allows for interplay between neutralino enhancement via axino production and decay, while simultaneously allowing for neutralino production and dilution via saxion production and decay, 3. it includes the effect of gravitino production and decay (not a big effect for the parameters presented here) and 4. it moves out of the "sudden decay" approximation and allows for continuous axino, saxion and gravitino decay. Our calculation allows for the accurate estimate of mixed axion/neutralino abundance for general choices of PQMSSM parameters. 


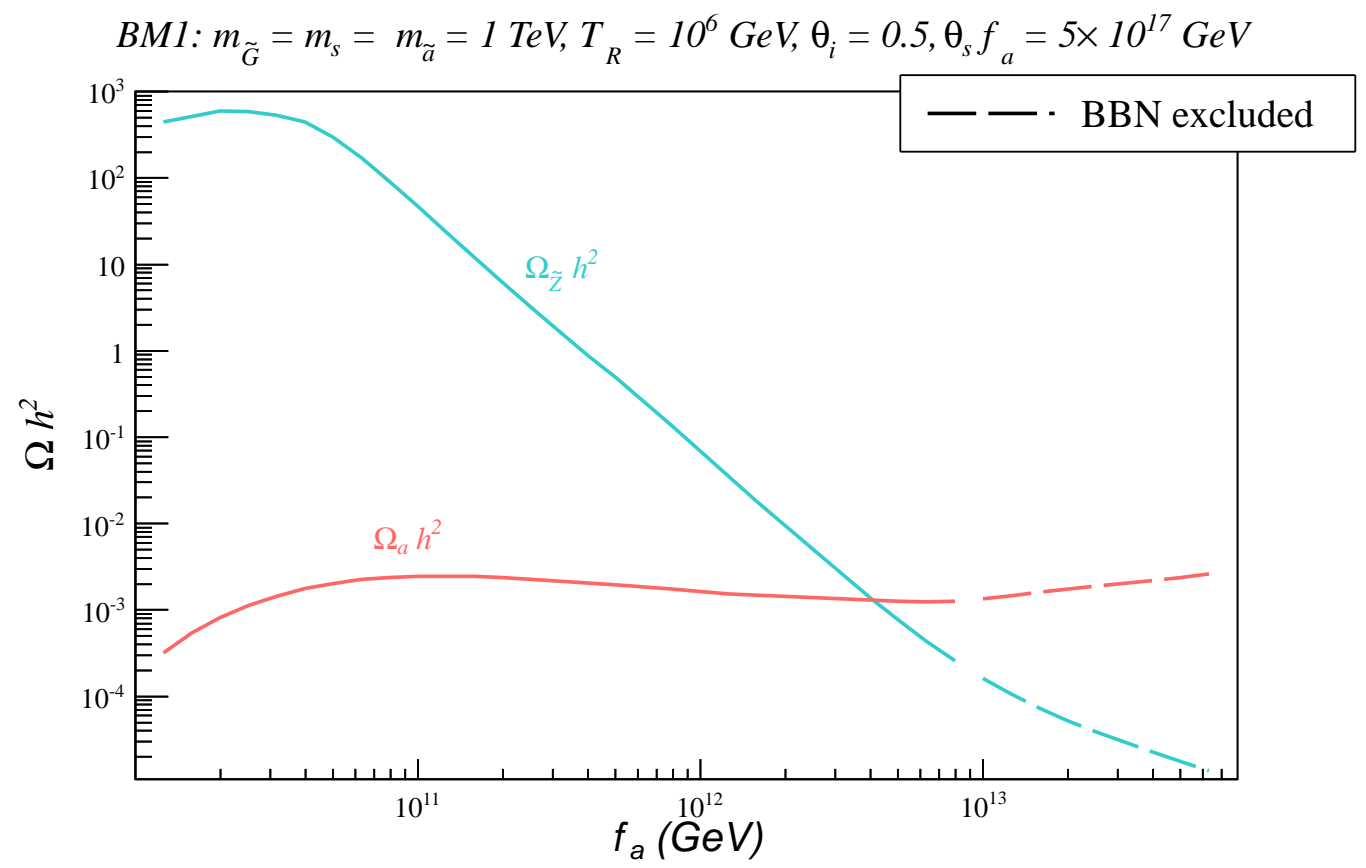

Figure 12: Calculated neutralino and axion abundance versus $f_{a}$ for SUSY model BM1 with $\theta_{s} f_{a}$ fixed at $5 \times 10^{17} \mathrm{GeV}$, and with $m_{\widetilde{G}}=m_{s}=m_{\tilde{a}}=1 \mathrm{TeV}, T_{R}=10^{6} \mathrm{GeV}$ and $\theta_{i}=0.5$.

In most gravity-mediated SUSY breaking models with gaugino mass unification, it is typically the case that the lightest SUSY particle is a bino-like neutralino. Over most of parameter space of models such as mSUGRA, bino-like neutralinos give rise to a dark matter abundance far above WMAP limits 19], and hence vast regions of parameter space are considered as excluded due to overproduction of neutralino dark matter. In this paper, we have shown that if the MSSM is extended to the PQMSSM- including an axion/saxion/axino supermultiplet- then SUSY models with a standard overabundance of neutralinos are typically still excluded, even for very large values of $f_{a} \lesssim 10^{14}-10^{15} \mathrm{GeV}$, where it might be expected that a high rate of entropy production from saxion decay would dilute the DM abundance. Here, we find that $s \rightarrow \tilde{g} \tilde{g}$ compensates against entropy dilution, and prevents the neutralino abundance from dropping into the measured range, unless the saxion decays are in violation of BBN bounds on late-decaying neutral particles. As noted earlier, our conclusion depends on at least three assumptions. First, we implemented the standard thermal axino production rates as calculated in the Ref's [21, 29, 30]. These rates should apply in supersymmetric versions of the KSVZ model where $P Q$-charged matter multiplets $\hat{\Phi}$ exist at or around the PQ breaking scale $f_{a}$. In a recent publication 61], it has been shown that in the SUSY DFSZ model, thermal axino production rates can be enhanced or diminished compared to their KSVZ values depending on PQMSSM parameters. Secondly, we assumed that saxion decay is dominated by two-body modes into gluon and gluino pairs. In the DFSZ model, decays into Higgs pairs or aa may also contribute, and even dominate the saxion decay modes. Thirdly, we have assumed saxion field strength $s(x) \equiv \theta_{s} f_{a}$ is of 
order the PQ-breaking scale $f_{a}$, i.e. that $\theta_{s} \sim 1$. We have also shown in Sec. 4 that if $\theta_{s} \gg 1$ and $m_{s}<2 m_{\tilde{g}}$, then CO-produced saxions can dominate the universe and dilute all thermal relics while avoiding BBN constraints.

In the case of a standard underabundance of neutralino CDM, a wide range of $f_{a}$ values are permitted, and can augment the neutralino DM into the measured range. In cases where the neutralinos still maintain an underabundance, the remaining abundance can be accommodated by axions. In these cases of a standard underabundance of neutralino $\mathrm{DM}$, the PQ scale $f_{a}$ can be pushed into the $10^{14}-10^{15} \mathrm{GeV}$ range, which is closer to expectations from string theory. For the case of very high $f_{a}$, then we typically expect the DM to be neutralino rather than axion dominated, since the neutralino abundance cannot be suppressed too much without violating BBN constraints.

\section{Acknowledgments}

This research was supported in part by the U.S. Department of Energy, by the Fulbright Program, CAPES and FAPESP.

\section{A. Boltzmann Equations for the PQMSSM}

As discussed in Sec. 2, we assume the following set of coupled differential equations:

$$
\begin{aligned}
\dot{n}_{i} & =-3 H n_{i}-\Gamma_{i} m_{i} \frac{n_{i}^{2}}{\rho_{i}}+\left[\left(n_{i}^{e q}(T)\right)^{2}-n_{i}^{2}\right]\langle\sigma v\rangle_{i}+\sum_{j} B R(j, i) \Gamma_{j} m_{j} \frac{n_{j}^{2}}{\rho_{j}} \\
\dot{S} & =\frac{R^{3}}{T} \sum_{i} B R(i, X) \Gamma_{i} m_{i} n_{i}
\end{aligned}
$$

with $H$ given by:

$$
H=\frac{1}{R} \frac{d R}{d t}=\sqrt{\frac{\rho_{T}}{3 M_{P}^{2}}},
$$

where $\rho_{T}$ is the total energy density.

In order to simplify the above equations we define:

$$
x=\ln \left(R / R_{0}\right), \quad N_{i}=\ln \left(n_{i} / s_{0}\right), \text { and } N_{S}=\ln \left(S / S_{0}\right)
$$

so we can write Eq's. A.1 as:

$$
\begin{aligned}
& N_{S}^{\prime}=\frac{1}{H T} \sum_{i} B R(i, X) \Gamma_{i} m_{i} \exp \left[N_{i}+3 x-N_{S}\right] \\
& N_{i}^{\prime}=-3-\frac{\Gamma_{i}}{H} \frac{m_{i}}{\rho_{i} / n_{i}}+\sum_{j \neq i} B R(j, i) \frac{\Gamma_{j}}{H} \frac{m_{j}}{\rho_{j} / n_{j}} \frac{n_{j}}{n_{i}}+\frac{\langle\sigma v\rangle_{i}}{H} n_{i}\left[\left(\frac{n_{i}^{e q}}{n_{i}}\right)^{2}-1\right]
\end{aligned}
$$

where $^{\prime}=d / d x$ and $n_{i}$ is given by $n_{i}=s_{0} e^{N_{i}}$.

The above equation for $N_{i}$ also applies for coherent oscillating fields, if we define:

$$
N_{i}=\ln \left(n_{i} / s_{0}\right), \text { and } n_{i} \equiv \rho_{i} / m_{i}
$$


SO

$$
N_{i}^{\prime}=-3-\frac{\Gamma_{i}}{H}
$$

where we assume that the coherent oscillating component does not couple to any of the other fields.

Since $H$ depends on the energy densities, to solve the above equations we must compute $\rho_{i}$ from $n_{i}$. However, even for particles following a thermal distribution, the energy density for each component cannot be directly obtained from $n_{i}$, unless the chemical potential $\left(\mu_{i}\right)$ is also given. Nonetheless, $\mu_{i}(T)$ is usually small in the relativistic regime, while in the non-relativistic regime we always have $\rho_{i}=m_{i} n_{i}$. Therefore, assuming that the fields follow a thermal distribution, a good approximation for $\rho_{i}$ as a function of $n_{i}$ is given by:

$$
\rho_{i}=n_{i} \times \begin{cases}m_{i} & , \text { if } T_{i}<m_{i} / 10 \\ m_{i} \frac{K_{1}\left(m_{i} / T_{i}\right)}{K_{2}\left(m_{i} / T_{i}\right)}+3 T_{i} & , \text { if } m_{i} / 10<T_{i}<3 m_{i} / 2 \\ N_{F} \frac{\pi^{4}}{\xi(3)} \frac{T_{i}}{30} & , \text { if } 3 m_{i} / 2<T_{i}\end{cases}
$$

where the modified Bessel functions, $K_{1}$ and $K_{2}$, are necessary to describe a smooth relativistic/non-relativistic transition and $N_{F}=1(7 / 6)$ for bosons (fermions).

The only remaining piece of information necessary for computing $\rho_{i}$ and $H$ and solving the Boltzmann equations is the definition of temperature for each component. The radiation temperature can be directly obtained from $N_{S}$ and $x$ :

$$
T=\left(\frac{g_{*}\left(T_{R}\right)}{g_{*}(T)}\right)^{1 / 3} T_{R} \exp \left[N_{S} / 3-x\right]
$$

For thermal fluids in equilibrium we always have $T_{i}=T$, but once they decouple, this is no longer true. However, the temperature of relativistic fluids scales as $T \propto R^{-1}$, while non-relativistic fluids have $T \propto R^{-2}$. Thus, we approximate $T_{i}$ by

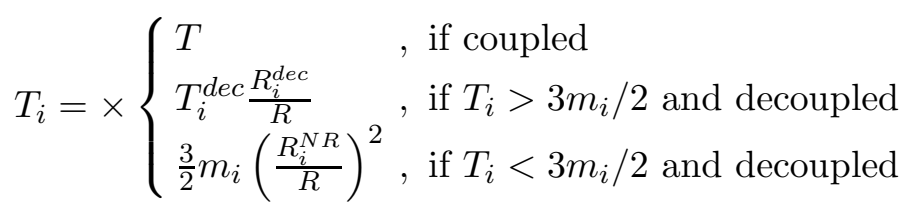

where $T_{i}^{d e c}, R_{i}^{\text {dec }}$ and $R_{i}^{N R}$ are the decoupling (freeze-out) temperature, the scale factor at freeze-out and the scale factor at the non-relativistic transition $\left(T_{i}=3 m_{i} / 2\right)$, respectively. If the fluid was never in thermal equilibrium, we take $T_{i}^{d e c}=T_{R}$. For coherent oscillating fluids we always have $T_{i}=0^{7}$.

Eq's. A.4 and A.5, with the auxiliary equations for $H$ (Eq. A.2), $\rho_{i}$ (Eq. A.8) and $T_{i}$ (Eq. A.10) form a set of closed equations, which can be solved once the initial conditions for the number densities $\left(n_{i}\right)$ and entropy $(S)$ are given. The initial entropy $S_{0}$ is trivially obtained, once we assume a radiation dominated universe at $T=T_{R}$ :

$$
S\left(T_{R}\right)=\frac{2 \pi^{2}}{45} g_{*}\left(T_{R}\right) T_{R}^{3} R_{0}^{3}
$$

\footnotetext{
${ }^{7}$ In principle, the approximations in Eq's. A.8 and A.10 can be avoided if we include equations for the chemical potentials $\mu_{i}(T)$. However, for simplicity, we use Eq's. A.8 and A.10 instead.
} 
For thermal fluids we take the initial number density as

$$
n_{i}\left(T_{R}\right)=\left\{\begin{array}{ll}
0 & , \text { if }\langle\sigma v\rangle_{i} n_{i}^{e q} /\left.H\right|_{T=T_{R}}<10 \\
n_{i}^{e q}\left(T_{R}\right), & \text { if }\langle\sigma v\rangle_{i} n_{i}^{e q} /\left.H\right|_{T=T_{R}}>10
\end{array},\right.
$$

while for coherent oscillating fluids the initial condition is set at the beginning of oscillations:

$$
n_{i}\left(T_{i}^{o s c}\right)=\frac{\rho_{i}^{0}}{m_{i}\left(T_{i}^{o s c}\right)}
$$

where $T_{i}^{o s c}$ is the oscillation temperature, given by $3 H\left(T_{i}^{o s c}\right)=m_{i}\left(T_{i}^{o s c}\right)$ and $\rho_{i}^{0}$ the initial energy density for oscillations. For the oscillating saxion and axion [39] fields the initial energy densities are given by:

$$
\begin{aligned}
& \rho_{a}^{0}=1.44 \frac{m_{a}(T)^{2}\left(f_{a}\right)^{2} \theta_{i}^{2}}{2} f\left(\theta_{i}\right)^{7 / 6} \\
& \rho_{s}^{0}=\min \left[2.1 \times 10^{-9}\left(\frac{2 \pi^{2} g_{*}\left(T_{R}\right) T_{R}^{3}}{45}\right)\left(\frac{T_{R}}{10^{5}}\right)\left(\frac{\theta_{s}\left(f_{a}\right)}{10^{12}}\right)^{2}, \frac{m_{s}^{2} \theta_{s}^{2}\left(f_{a}\right)^{2}}{2}\right]
\end{aligned}
$$

where $f\left(\theta_{i}\right)=\ln \left[e /\left(1-\theta_{i}^{2} / \pi^{2}\right)\right]$ and $\theta_{i} f_{a}$ and $\theta_{s} f_{a}$ are the initial axion and saxion field amplitudes. The definition of $\rho_{s}^{0}$ accounts for the possibility of saxion oscillations beginning during inflation (if $T_{R}<T_{o s c}$ ).

In order to compute the source term in Eq. A.1, we must specify the annihilation cross-sections $\langle\sigma v\rangle_{i}$, the branching ratios $B R(i, j)$ and $B R(i, X)$ and the the decay widths $\Gamma_{i}$. The annihilation cross-sections for axions, saxions, axinos and gravitinos are given by the expressions [29, 30, 62]

$$
\begin{aligned}
\langle\sigma v\rangle_{a} & =10^{-4} \frac{g_{s}^{6}}{\left(f_{a}\right)^{2}}\left[4.19 \ln \left(1.5 / g_{s}^{2}\right)+1.68 \times \theta\left(T-m_{a}(T)\right)\right] \\
\langle\sigma v\rangle_{\tilde{a}} & =10^{-5} \frac{g_{s}^{6}}{\left(f_{a}\right)^{2}}\left[3+3.87 \times \theta\left(T-m_{\tilde{a}}\right)\left\{\begin{array}{l}
23.863 g_{s}^{-0.7}-0.784 \quad, \text { if } g_{s}>0.35 \\
4.47+31 \ln \left(1.4 / g_{s}\right)-0.784, \text { if } g_{s}<0.35
\end{array}\right]\right. \\
\langle\sigma v\rangle_{\widetilde{G}} & =\frac{1.37}{M_{P}^{2}} \times\left[72 g_{s}^{2} \ln \left(1.271 / g_{s}\right)\left(1+\frac{M_{3}^{2}}{3 m_{\widetilde{G}}^{2}}\right)\right. \\
& \left.+27 g^{2} \ln (1.312 / g)\left(1+\frac{M_{2}^{2}}{3 m_{\widetilde{G}}^{2}}\right)+11 g^{\prime 2} \ln \left(1.266 / g^{\prime}\right)\left(1+\frac{M_{1}^{2}}{3 m_{\widetilde{G}}^{2}}\right)\right]
\end{aligned}
$$

while $\langle\sigma v\rangle_{\widetilde{Z}_{1}}(T)$ is extracted from IsaReD [38]. The second term in the expressions for $\langle\sigma v\rangle_{a}$ and $\langle\sigma v\rangle_{\tilde{a}}$ represent contributions from $1 \rightarrow 2$ decays of particles with thermal masses. Therefore, these terms should not be included unless $T>m_{a, \tilde{a}}$, as indicated by the $\theta$ functions above. The expression for the axino effective cross-section is set to reproduce the numerical results in [30]. Since the saxion thermal production has not been computed, we approximate it by the axion expression:

$$
\langle\sigma v\rangle_{s}=\langle\sigma v\rangle_{a}
$$

with $m_{a} \rightarrow m_{s}$. 
For obtaining the various unstable particle widths, we calculate $\Gamma_{\tilde{a}}$ from the $\tilde{a} \rightarrow \tilde{g} g$, $\widetilde{Z}_{i} \gamma$ and $\widetilde{Z}_{i} Z$ partial widths as presented in Ref. [26]. For gravitino decays, we adopt the gravitino widths as presented in Ref. [33]. For the saxion width, we include $\Gamma_{s}$ from the $s \rightarrow g g$ and $s \rightarrow \tilde{g} \tilde{g}$ decays as presented in Ref. [53]. We note here that in the DFSZ model, it is also possible to have $s \rightarrow h$ decays and possibly $s \rightarrow a a$ decays. We neglect these latter two cases, so that our results apply to the supersymmetrized KSVZ model, where the $g g$ and $\tilde{g} \tilde{g}$ final states should dominate.

Once the total and partial widths are known, we can easily compute the required branching ratios:

$$
B R\left(\tilde{a}, \widetilde{Z}_{1}\right)=1, B R\left(s, \widetilde{Z}_{1}\right)=2 \times \frac{\Gamma(s \rightarrow \tilde{g} \tilde{g})}{\Gamma_{s}}, B R\left(\widetilde{G}, \widetilde{Z}_{1}\right)=1
$$

The factor 2 in $B R\left(s, \widetilde{Z}_{1}\right)$ takes care of the multiplicity of neutralinos for each saxion cascade decay. While the $s \rightarrow g g$ decay width is always dominant, we showed in Sec. 3 that $s \rightarrow \tilde{g} \tilde{g}$ plays a crucial role in the PQMSSM dark matter cosmology.

Finally, we assume that the branching ratios for computing the energy injection into the thermal bath from unstable particle decays are given by:

$$
B R(\tilde{a}, X)=B R(s, X)=B R(\widetilde{G}, X)=1
$$

Although some of the decay energy is lost into neutralinos (except for $s \rightarrow g g$ decays), we assume that in the final product of the cascade decay of axinos, saxions and gravitinos most of the initial energy has been converted into radiation, so Eq. A.16 consists in a good approximation.

\section{References}

[1] For a review, see R. D. Peccei, Lect. Notes Phys. 741 (2008) 3 [arXiv:hep-ph/0607268].

[2] H. Baer and X. Tata, Weak Scale Supersymmetry: From Superfields to Scattering Events, (Cambridge University Press, 2006).

[3] S. Dimopoulos, S. Raby and F. Wilczek, Phys. Rev. D 24 (1981) 1681; U. Amaldi, W. de Boer and H. Furstenau, Phys. Lett. B 260 (1991) 447; J. Ellis, S. Kelley and D. V. Nanopoulos, Phys. Lett. B 260 (1991) 131; P. Langacker and Luo, Phys. Rev. D 44 (1991) 817 .

[4] S. Heinemeyer, W. Hollik, D. Stockinger, A. M. Weber and G. Weiglein, J. High Energy Phys. 0608 (2006) 052.

[5] R. Peccei and H. Quinn, Phys. Rev. Lett. 38 (1977) 1440 and Phys. Rev. D 16 (1977) 1791; S. Weinberg, Phys. Rev. Lett. 40 (1978) 223; F. Wilczek, Phys. Rev. Lett. 40 (1978) 279.

[6] J. E. Kim, Phys. Rev. Lett. 43 (1979) 103; M. A. Shifman, A. Vainstein and V. I. Zakharov, Nucl. Phys. B 166 (1980) 493.

[7] M. Dine, W. Fischler and M. Srednicki, Phys. Lett. B 104 (1981) 199; A. P. Zhitnitskii, Sov. J. Nucl. 31 (1980) 260. 
[8] For a recent review, see P. Sikivie, hep-ph/0509198; M. Turner, Phys. Rept. 197 (1990) 67; J. E. Kim, Phys. Rept. 150 (1987) 1; J. E. Kim and G. Carosi, Rev. Mod. Phys. 82 (2010) 557.

[9] For recent reviews of axino dark matter, see F. Steffen, Eur. Phys. J. C 59 (2009) 557; L. Covi and J. E. Kim, New J. Phys. 11 (2009) 105003.

[10] D. Dicus, E. Kolb, V. Teplitz and R. Wagoner, Phys. Rev. D 18 (1978) 1829 and Phys. Rev. D 22 (1980) 839; for a review, see G. Raffeldt, hep-ph/0611350.

[11] L. F. Abbott and P. Sikivie, Phys. Lett. B 120 (1983) 133; J. Preskill, M. Wise and F. Wilczek, Phys. Lett. B 120 (1983) 127; M. Dine and W. Fischler, Phys. Lett. B 120 (1983) 137; M. Turner, Phys. Rev. D 33 (1986) 889.

[12] P. Sikivie, Phys. Lett. B 695 (2011) 22; O. Erken, P. Sikivie, H. Tam and Q. Yang, arXiv:1104.4507 (2011).

[13] E. J. Chun, H. B. Kim and D. H. Lyth, Phys. Rev. D 62 (2000) 125001; E. J. Chun, H. B. Kim, K. Kohri and D. H. Lyth, J. High Energy Phys. 0803 (2008) 061; S. Kim, W. I. Park and E. D. Stewart, J. High Energy Phys. 0807 (2009) 015.

[14] T. Asaka, K. Ishiwata and T. Moroi, Phys. Rev. D 73 (2006) 051301 and Phys. Rev. D 75 (2007) 065001.

[15] M. Kawasaki, K. Kohri, T. Moroi and A. Yotsuyanagi, Phys. Rev. D 78 (2008) 065011.

[16] M. Gell-Mann, P. Ramond and R. Slansky, in Supergravity, Proceedings of the Workshop, Stony Brook, NY 1979 (North-Holland, Amsterdam); T. Yanagida, KEK Report No. 79-18, 1979; R. Mohapatra and G. Senjanovic, Phys. Rev. Lett. 44 (1980) 912.

[17] J. Ellis and K. Olive, Phys. Lett. B 514 (2001) 114; H. Baer and A. Box, Eur. Phys. J. C 68 (2010) 523 .

[18] E. Komatsu et al. (WMAP collaboration), arXiv:1001.4538 (2010).

[19] H. Baer, A. Box and H. Summy, J. High Energy Phys. 1010 (2010) 023.

[20] K. Rajagopal, M. Turner and F. Wilczek, Nucl. Phys. B 358 (1991) 447.

[21] L. Covi, J. E. Kim and L. Roszkowski, Phys. Rev. Lett. 82 (1999) 4180; L. Covi, H. B. Kim, J. E. Kim and L. Roszkowski, J. High Energy Phys. 0105 (2001) 033.

[22] H. Baer, A. Box and H. Summy, J. High Energy Phys. 0908 (2009) 080.

[23] P. Moxhay and K. Yamamoto, Phys. Lett. B 151 (1985) 363; E. Chun and A. Lukas, Phys. Lett. B 357 (1995) 43.

[24] K-Y. Choi, J. E. Kim, H. M. Lee and O. Seto, Phys. Rev. D 77 (2008) 123501.

[25] For a review, see G. Jungman, K. Griest, M. Kamionkowski, Phys. Rept. 267 (1996) 195.

[26] H. Baer, A. Lessa, S. Rajagopalan and W. Sreethawong, JCAP1106 (2011) 031.

[27] M. Turner, Phys. Rev. D 28 (1983) 1243.

[28] K. J. Bae, K. Choi and S. H. Im, J. High Energy Phys. 1108 (2011) 065.

[29] P. Graf and F. Steffen, Phys. Rev. D 83 (2011) 070511.

[30] A. Strumia, J. High Energy Phys. 1006 (2010) 036.

[31] P. Svrcek and E. Witten, J. High Energy Phys. 0606 (2006) 051. 
[32] E. Kolb and M. Turner, The Early Universe, Addison-Wesley Pub. (1990).

[33] K. Kohri, T. Moroi and A. Yotsuyanagi, Phys. Rev. D 73 (2006) 123511.

[34] R. H. Cyburt, J. Ellis, B. D. Fields and K. A. Olive, Phys. Rev. D 67 (2003) 103521; R. H. Cyburt, J. Ellis, B. D. Fields, F. Luo, K. Olive and V. Spanos, JCAP0910 (2009) 021.

[35] M. Kawasaki, K. Kohri and T. Moroi, Phys. Lett. B 625 (2005) 7 and Phys. Rev. D 71 (2005) 083502; K. Kohri, T. Moroi and A. Yotsuyanagi, Phys. Rev. D 73 (2006) 123511; for an update, see M. Kawasaki, K. Kohri, T. Moroi and A. Yotsuyanagi, Phys. Rev. D 78 (2008) 065011.

[36] K. Jedamzik, Phys. Rev. D 70 (2004) 063524 and Phys. Rev. D 74 (2006) 103509.

[37] ISAJET, by H. Baer, F. Paige, S. Protopopescu and X. Tata, hep-ph/0312045; see also H. Baer, J. Ferrandis, S. Kraml and W. Porod, Phys. Rev. D 73 (2006) 015010.

[38] H. Baer, C. Balazs and A.Belyaev, J. High Energy Phys. 0203 (2002) 042.

[39] L. Visinelli and P. Gondolo, Phys. Rev. D 80 (2009) 035024.

[40] M. Drees and M. Nojiri, Phys. Rev. D 47 (1993) 376; H. Baer and M. Brhlik, Phys. Rev. D 57 (1998) 567; H. Baer, M. Brhlik, M. Diaz, J. Ferrandis, P. Mercadante, P. Quintana and X. Tata, Phys. Rev. D 63 (2001) 015007; J. Ellis, T. Falk, G. Ganis, K. Olive and M. Srednicki, Phys. Lett. B 510 (2001) 236; L. Roszkowski, R. Ruiz de Austri and T. Nihei, J. High Energy Phys. 0108 (2001) 024; A. Djouadi, M. Drees and J. L. Kneur, J. High Energy Phys. 0108 (2001) 055; A. Lahanas and V. Spanos, Eur. Phys. J. C 23 (2002) 185.

[41] L. Randall and R. Sundrum, Nucl. Phys. B 557 (1999) 79; G. Giudice, M. Luty, H. Murayama and R. Rattazzi, J. High Energy Phys. 12 (1998) 027.

[42] H. Baer, S. de Alwis, K. Givens, S. Rajagopalan and H. Summy, J. High Energy Phys. 1005 (2010) 069 .

[43] G. Lazarides, C. Panagiotakapolous and Q. Shafi, Phys. Lett. B 192 (1987) 323; G. Lazarides, R. Schaefer, D. Seckel and Q. Shafi, Nucl. Phys. B 346 (1990) 193; J. McDonald, Phys. Rev. D 43 (1991) 1063; C. Pallis, Astropart. Phys. 21 (2004) 689.

[44] J. E. Kim, Phys. Rev. Lett. 67 (1991) 3465.

[45] M. Kawasaki, T. Moroi and T. Yanagida, Phys. Lett. B 383 (1996) 313.

[46] K. Choi, E. J. Chun and J. E. Kim, Phys. Lett. B 403 (1997) 209.

[47] T. Banks, M. Dine and M. Graesser, Phys. Rev. D 68 (2003) 075011.

[48] P. Fox, A. Pierce and S. Thomas, hep-th/0409059 (2004).

[49] B. Acharya, K. Bobkov and P. Kumar, J. High Energy Phys. 1011 (2010) 105.

[50] J. Hasenkamp and J. Kersten, Phys. Rev. D 82 (2010) 115029.

[51] M. Kawasaki, N. Kitajima and K. Nakayama, Phys. Rev. D 83 (2011) 123521.

[52] H. Baer and A. Lessa, J. High Energy Phys. 1106 (2011) 027.

[53] H. Baer, S. Kraml, A. Lessa and S. Sekmen, JCAP1104 (2011) 039.

[54] M. Kawasaki, K. Nakayama and M. Senami, JCAP0803 (2008) 009. 
[55] K. L. Chan, U. Chattopadhyay and P. Nath, Phys. Rev. D 58 (1998) 096004; J. Feng, K. Matchev and T. Moroi, Phys. Rev. Lett. 84 (2000) 2322 and Phys. Rev. D 61 (2000) 075005; J. Feng, K. Matchev and F. Wilczek, Phys. Lett. B 482 (2000) 388; J. Feng and F. Wilczek, Phys. Lett. B 631 (2005) 170; see also H. Baer, C. H. Chen, F. Paige and X. Tata, Phys. Rev. D 52 (1995) 2746 and Phys. Rev. D 53 (1996) 6241; H. Baer, C. H. Chen, M. Drees, F. Paige and X. Tata, Phys. Rev. D 59 (1999) 055014; for a model-independent approach, see H. Baer, T. Krupovnickas, S. Profumo and P. Ullio, J. High Energy Phys. $0510(2005) 020$.

[56] H. Baer and J. O'Farrill, JCAP 0404 (2004) 005; H. Baer, A. Belyaev, T. Krupovnickas and J. O'Farrill, JCAP 0408 (2004) 005.

[57] T. Moroi and L. Randall, Nucl. Phys. B 570 (2000) 455.

[58] G. Gelmini and P. Gondolo, Phys. Rev. D 74 (2006) 023510; G. Gelmini, P. Gondolo, A. Soldatenko and C. Yaguna, Phys. Rev. D 74 (2006) 083514; G. Gelmini, P. Gondolo, A. Soldatenko and C. Yaguna, Phys. Rev. D 76 (2007) 015010.

[59] B. Acharya, K. Bobkov, G. Kane, P. Kumar and J. Shao, Phys. Rev. D 76 (2007) 126010 and Phys. Rev. D 78 (2008) 065038; B. Acharya, P. Kumar, K. Bobkov, G. Kane, J. Shao and S. Watson, J. High Energy Phys. 0806 (2008) 064.

[60] H. Baer, R. Dermisek, S. Rajagopalan and H. Summy, JCAP1007 (2010) 014.

[61] K. Y. Choi, L. Covi, J. E. Kim and L. Roszkowski, arXiv:1108.2282 (2011).

[62] J. Pradler and F. Steffen, Phys. Lett. B 648 (2007) 224; see also M. Bolz, A. Brandenburg and W. Buchmuller, Nucl. Phys. B 606 (2001) 518; J. Pradler and F. Steffen, Phys. Rev. D 75 (2007) 023509; V. S. Rychkov and A. Strumia, Phys. Rev. D 75 (2007) 075011. 